\title{
Theoretical study and numerical simulation of pattern formation in the deterministic and stochastic Gray-Scott equations*
}

\author{
Erika Hausenblas ${ }^{\mathrm{a}, *}$, Tsiry Avisoa Randrianasolo ${ }^{\mathrm{b}}$, Mechtild Thalhammer $^{\mathrm{c}}$ \\ ${ }^{a}$ Department of Mathematics and Information Technology, Montanuniversität Leoben, 8700 Leoben, Austria \\ ${ }^{b}$ Faculty of Mathematics, Bielefeld University, 33615 Bielefeld, Germany \\ ${ }^{c}$ Institut für Mathematik, Leopold-Franzens-Universität Innsbruck, 6020 Innsbruck, Austria
}

\begin{abstract}
Mathematical models based on systems of reaction-diffusion equations provide fundamental tools for the description and investigation of various processes in biology, biochemistry, and chemistry; in specific situations, an appealing characteristic of the arising nonlinear partial differential equations is the formation of patterns, reminiscent of those found in nature. The deterministic Gray-Scott equations constitute an elementary two-component system that describes autocatalytic reaction processes; depending on the choice of the specific parameters, complex patterns of spirals, waves, stripes, or spots appear.

In the derivation of a macroscopic model such as the deterministic Gray-Scott equations from basic physical principles, certain aspects of microscopic dynamics, e.g. fluctuations of molecules, are disregarded; an expedient mathematical approach that accounts for significant microscopic effects relies on the incorporation of stochastic processes and the consideration of stochastic partial differential equations.

The present work is concerned with a theoretical and numerical study of the stochastic GrayScott equations driven by independent spatially time-homogeneous Wiener processes. Under suitable regularity assumptions on the prescribed initial states, existence, as well as the uniqueness of the solution processes, is proven. Numerical simulations based on the application of a time-adaptive first-order operator splitting method and the fast Fourier transform illustrate the formation of patterns in the deterministic case and their variation under the influence of stochastic noise.
\end{abstract}

Keywords: Mathematical biology, Reaction-diffusion systems, Turing patterns, Stochastic partial differential equations, Operator splitting method, Numerical approximation

*This work was supported by the Austrian Science Fund (FWF): P25968 and the German research foundation as part of the SFB 1283.

* Corresponding author

Email address: erika.hausenblas@unileoben.ac.at (Erika Hausenblas) 
2000 MSC: 60H15, 92B05, 92F05, 35G50, 35Q92, 60G57

\section{Introduction}

This work is concerned with the theoretical study and numerical simulation of the stochastic Gray-Scott equations, which constitute a two-component system of reaction-diffusion equations driven by a spatial time homogenous Wiener process. Despite its comparatively simple structure, the underlying system of deterministic nonlinear partial differential equations exhibits a large variety of complex patterns for different choices of the specific parameters.

Biochemical and chemical kinetics reactions have been a rich source for the observation of spatial-temporal patterns; the derivation and investigation of suitable mathematical models for such phenomena remain a challenging question.

A famous example of non-equilibrium thermodynamics is the Belousov-Zhabotinsky reaction, discovered by BoRIS BELOUSOV at the beginning of the 1950s; he succeeded in stimulating a reaction of chemical substances that led to periodic changes of their concentrations, visible as oscillations in color.

An elementary mathematical model for this kind of nonlinear chemical oscillators is the Brusselator, a system of reaction-diffusion equations proposed by Prigogine, Lefever [28, Eq. (3.6)]; in a dimensionless formulation, the considered system of nonlinear partial differential equations has the structure

$$
\left\{\begin{array}{l}
\partial_{t} u(x, t)=r_{u} \Delta u(x, t)+h_{u}(u(x, t), v(x, t)), \\
\partial_{t} v(x, t)=r_{v} \Delta v(x, t)+h_{v}(u(x, t), v(x, t)),
\end{array}\right.
$$

where the real-valued space-time-dependent functions $u, v: I \times[0, T] \subset \mathbb{R}^{d} \times \mathbb{R} \rightarrow \mathbb{R}$ are associated with the concentrations of the chemical substances, $\Delta$ represents the Laplacian with respect to the spatial variables, the constants $r_{u}, r_{v}>0$ denote the diffusion coefficients, and the nonlinear functions $h_{u}, h_{v}: \mathbb{R}^{2} \rightarrow \mathbb{R}$ describe the reactions.

ALAN TURING suggested that the main mechanisms of morphogenesis are captured by mathematical models for systems of chemical substances, which react together and diffuse through tissue. In a seminal work [49], he studies reaction-diffusion equations that have a similar form as 1.1a) on different geometries of the domain, amongst others on spheres and rings, and explains the development of patterns from almost uniform initial states by instabilities of homogeneous equilibria; we refer to such patterns as Turing patterns.

In the present work, we focus on a classical mathematical model for isothermal autocatalytic reaction processes that goes back to GraY, ScOtT [18, 19, 20, 21]; depending on the choice of the feed and removal rates of the reactants, Turing patterns of spirals, waves, stripes, or spots appear. The deterministic Gray-Scott equations are cast into the form 1.1a with cubic 
reaction terms

$$
\begin{gathered}
h_{u}(u, v)=\beta_{u}(1-u)-g(u, v), \quad h_{v}(u, v)=-\beta_{v} v+g(u, v), \\
g(u, v)=u v^{2},
\end{gathered}
$$

involving certain constants $\beta_{u}, \beta_{v}>0$.

Related systems of reaction-diffusion equations are also studied in other contexts. KIERSTEAD, Slobodkin 25] describe the survival of phytoplankton populations in body of water. SEGEL, JACKSON 42] consider predator-prey interaction models with diffusion; based on a linear stability analysis, they demonstrate that spatially uniform equilibria which are stable for homogeneously distributed populations become unstable through dispersal effects. LEVIN, SEGEL [29] study the dynamics of plankton populations. KLAUsmeIER [26] discusses a model for semi-arid ecosystems on sloped terrains. MURRAY [35, 36] describes coat patterns in animal tails; numerical simulations on surfaces with periodic and homogeneous Neumann boundary conditions, respectively, show patterns of stripes and spots that are similar to the markings observed on the tails of felines.

Reaction-diffusion systems like (1.1a) constitute prevalent macroscopic models for microscopic phenomena; however, as their derivation relies on fundamental balance laws and Fick's law of diffusion, significant aspects of microscopic dynamics such as fluctuations of molecules are disregarded. An appropriate mathematical approach to establish more realistic models is the incorporation of a random noise mimicking these fluctuations.

BiAnCALANi et al. 9] introduce a microscopic model of the Brusselator that includes stochastic fluctuations. Compartment-based approaches use a division of the domain into certain compartments and a simulation of the number of molecules in each compartment; CAO, ERBAN [10] investigate the dependence of stochastic Turing patterns on the compartment size. In MCKANE et al. 34, it is shown how a stochastic amplification of a Turing instability gives rise to spatial-temporal patterns. Treatments of the stochastic Brusselator in different respects are found in [2, 41, 48].

The main theoretical contribution of this paper is to show (or derive) the existence and uniqueness of a solution for the Gray-Scott equations driven by independent spatially timehomogeneous Wiener processes. Besides, some numerical simulations are presented as an illustration. Here, the employed numerical approximation is based on a first-order operator splitting method and the fast Fourier transform; in order to enhance the reliability of the computations, we adapt the time stepsizes accordingly to the sizes of the nonlinear terms for particular realisations.

The theory of stochastic partial differential equations provides the basis of our investigations; for a comprehensive treatment of the fundamentals as well as an extensive bibliography, we refer to the monographs [12, 14, 30, 15].

This manuscript has the following structure. In Section 2, we introduce compact reformulations of the deterministic and stochastic Gray-Scott equations as well as the needed hypotheses 
on the driving Wiener processes and the initial states, subsequently, we state the main result ensuring the existence and uniqueness of the non-negative solution processes. In Section 3 , to complement our theoretical analysis, we present numerical simulations for the Gray-Scott equations in two space dimensions that illustrate the formation of patterns in the deterministic case and their variation under the influence of stochastic noise. Finally, in the Appendix we define the multiplication operator and summary the most important inequality.

\section{Stochastic Gray-Scott equations}

In this section, we state the mathematical formulation of the stochastic Gray-Scott equations, introduce the underlying spaces, review basic auxiliary results on spatially time homogeneous Wiener processes as well as associated stochastic integrals, and specify the hypotheses under which a solution exists. We begin with the mathematical formulation of the stochastic Gray-Scott equations. In the system, $u$ and $v$ are concentrations of two reactants $U$ and $V$, normalized as dimensionless units. The parameters $f$ and $k$ represent the feed rate and removal rate of the reactants. We recall that the parameters $r_{u}, r_{v}>0$ correspond to the diffusion coefficients. These parameters have a significant effect at the form of the observed patterns. The equation is given as follows

$$
\left\{\begin{aligned}
d u(t, x)= & \left(r_{u} \Delta u(t, x)-u(t, x) v^{2}(t, x)+f(1-u(t, x))\right) d t \\
& +\sigma_{u} u(t, x) \circ d W_{1}(t, x), \quad x \in I, t>0, \\
d v(t, x)=\left(r_{v} \Delta v(t, x)+u(t, x) v^{2}(t, x)-\right. & (f+k) v(t, x)) d t \\
& +\sigma_{v} v(t, x) \circ d W_{2}(t, x), \quad x \in I, t>0,
\end{aligned}\right.
$$

where $I=[0,1]^{d}$ be a bounded domain, $d=1,2, A=\Delta$ be the Laplace operator with periodic, or Dirichlet boundary conditions. The initial conditions are given by $u_{0}$ and $v_{0}$. Since the white noise is an approximation of a continuously fluctuating noise with finite memory being much shorter than the dynamical timescales, the representation of the stochastic integral as a Stratonovich stochastic integral is appropriate.

For suitable initial conditions and choices of the parameters, the formation of patterns is observed in the Gray-Scott equations. For convenience, we suppose that the constants that determine the strength of the multiplicative stochastic noise are positive, i.e. $\sigma_{u}, \sigma_{v} \geq 0$; evidently, the deterministic Gray-Scott equations (1.1) are retained from (2.1) for the special case $\left(\sigma_{u}, \sigma_{v}\right)=(0,0)$.

In this work, we focus on situations where the Gray-Scott equations (2.1) are driven by independent spatially time-homogeneous Wiener processes; as relevant concrete examples, we 
study the Gray Scott system driven by fractional Gaussian field. Let

$$
\mathfrak{A}=\left(\Omega, \mathcal{A},(\mathcal{A}(t))_{t \in[0, T]}, \mathbb{P}\right)
$$

be a complete probability space with associated filtration satisfying the standard assumptions; for our purposes, it suffices to consider a finite time interval. Let $\left\{\beta_{k}: k \in \mathbb{Z}^{d}\right\}$ be a family of one-dimensional standard Brownian motions defined over $\mathfrak{A}$. Here, we consider our equation on the $d$ dimensional torus. In the case of a single dimension, a complete orthonormal system of the underlying Lebesgue space $L^{2}(I):=L^{2}(I, \mathbb{R})$ is given by sine and cosine functions

$$
\psi_{m}(x)= \begin{cases}\sqrt{2} \sin (2 \pi m x) & \text { if } m \geq 1 \\ \sqrt{2} & \text { if } m=0 \\ \sqrt{2} \cos (\pi 2 m x) & \text { if } m \leq-1\end{cases}
$$

The extension to higher space dimensions relies on tensor products, i.e., for a multiindex $m=$ $\left(m_{1}, \ldots, m_{d}\right) \in \mathbb{Z}^{d}$ we have

$$
\phi_{m}(x)=\prod_{j=1}^{d} \phi_{m_{j}}\left(x_{j}\right), \quad x \in I .
$$

The corresponding eigenvalues are given by

$$
\lambda_{m}=-4 \pi^{2} \sum_{j=1}^{d} m_{j}^{2}, \quad m=\left(m_{1}, \ldots, m_{d}\right) \in \mathbb{Z}^{d} .
$$

The spatially time-homogenous Wiener process can be expressed in terms of the orthogonal system, i.e.,

$$
W(t, x)=\sum_{k \in \mathbb{Z}^{d}} \delta_{k} \psi_{k}(x) \beta_{k}(t)
$$

where the family $\left\{\beta_{k}: k \in \mathbb{Z}^{d}\right\}$ is a family of independent and identically distributed standard Brownian motions. For simplicity, we assume in our work that $\delta_{k}=\left(\alpha-D \lambda_{k}\right)^{-\gamma}, k \in \mathbb{Z}^{d}$, with certain positive constants adjusted in the numerical examples. Going back to our equation (2.1), we impose the following hypothesis.

Hypothesis 2.1. The Wiener processes $W_{1}$ and $W_{2}$ are spatially time-homogenous Wiener processes such that

$$
W_{j}(t, x)=\sum_{k \in \mathbb{Z}^{d}}\left(\alpha-D \lambda_{k}\right)^{-\gamma_{j}} \psi_{k}(x) \beta_{k}(t), \quad j=1,2
$$

with $\gamma_{j}>\frac{d}{2}$. 
In our case, Hypothesis 2.1 means that the sum defined by

$$
S(\gamma):=\sum_{k \in \mathbb{Z}^{d}}\left(\alpha-D \lambda_{k}\right)^{-2 \gamma}
$$

is bounded for $\gamma_{1}$ and $\gamma_{2}$. For simplicity we assume that $\gamma_{1}=\gamma_{2}=\gamma$. Since the solutions $u$ and $v$ of the Gray Scott system have to be non-negative, the initial conditions $u_{0}$ and $v_{0}$ have to be non-negative. Besides, we have to impose some regularity assumptions on the initial condition to get existence and uniqueness of the solution.

Hypothesis 2.2. Let $u_{0}, v_{0} \in L^{2}(I)$ such that

1. $u_{0} \geq 0$ and $v_{0} \geq 0$

2. $u_{0}$ and $v_{0}$ belong to $L^{6}(I)$, in particular we have $\mathbb{E}\left|u_{0}\right|_{L^{6}}^{6}<\infty$ and $\mathbb{E}\left|v_{0}\right|_{L^{6}}^{6}<\infty$.

3. $u_{0}$ and $v_{0}$ belong to $H_{4}^{1}(I)$, in particular $\mathbb{E}\left|u_{0}\right|_{H_{4}^{1}}^{4}<\infty$ and $\mathbb{E}\left|v_{0}\right|_{H_{4}^{1}}^{4}<\infty$.

In system (2.1) we interpreted the stochastic integral as a Stratonovich integral. White noise is an idealisation; real fluctuating forcing has a finite amplitude and a finite timescale; white noise is an idealisation of delta-function-correlated noise. If now the white noise is approximated by a continuously fluctuating noise with finite memory (much shorter than dynamical timescales), i.e., by noise with a finite correlation time $\tau$, and then the limit is taken for $\tau \rightarrow 0$, the Wong-Zakai Theorem gives as the appropriate representation of the white noise the Stratonovich integral. In this sense, the Startonovich integral models the natural one, the drawback is that the Stratonovich integral is not a martingale, and, therefore, the Itô isometry and Burkholder-Davis-Gundy inequality cannot be applied to the Stratonovich integral. Although here in the article we analyse a more general system, where the integral is interpreted as an Itô integral. To show that the system (2.1) has a unique solution, we first transform the system (2.1) into a system, where the integral can be interpreted in the Itô sense by adding a correction term, and, then, we show that the correction term behaves nicely. One can find a survey of some facts about the Stratonovich integral in Chapter 4.5.2 in [15]. In this way, it can be shown that the solution to (2.1) and the solution to

$$
\left\{\begin{aligned}
d u(t, x)= & \left(r_{u} \Delta u(t, x)-u(t, x) v^{2}(t, x)+f-\left(f-\sigma_{u} S\left(\gamma_{1}\right)\right) u(t, x)\right) d t \\
& +\sigma_{u} u(t, x) d W_{1}(t, x), \quad x \in I, t>0, \\
d v(t, x)= & \left(r_{v} \Delta v(t, x)+u(t, x) v^{2}(t, x)-\left(f+k-\sigma_{v} S\left(\gamma_{2}\right)\right) v(t, x)\right) d t \\
& +\sigma_{v} v(t, x) d W_{2}(t, x), \quad x \in I, t>0,
\end{aligned}\right.
$$


are equivalent. For simplicity we will combine the coefficient and consider the following system

$$
\left\{\begin{aligned}
& d u(t, x)=\left.\left(r_{u} \Delta u(t, x)-u(t, x) v^{2}(t, x)+\rho+\alpha_{u} u(t, x)\right)\right) d t \\
&+\sigma_{u} u(t, x) d W_{1}(t, x), \quad x \in I, t>0 \\
& d v(t, x)=\left(r_{v} \Delta v(t, x)+u(t, x) v^{2}(t, x)+\alpha_{v} v(t, x)\right) d t \\
&+\sigma_{v} v(t, x) d W_{2}(t, x), \quad x \in I, t>0
\end{aligned}\right.
$$

where $\rho, \alpha_{u}$ and $\alpha_{v}$ are real-valued number, not necessarily positive and the stochastic integral is interpreted in the sense of Itô. For this system we can show the following Theorem.

Theorem 2.1. Let us assume that $u_{0}, v_{0}$ are satisfying the Hypothesis 2.2 and the Wiener processes $W_{1}$ and $W_{2}$ the Hypothesis 2.1. Then there exists a couple of progressively measurable processes $(u, v)$ solving the system of equations (2.7) and for all $\delta<1 \mathbb{P}\left(u \in C\left(0, T ; H_{2}^{\delta}(I)\right)\right)=1$. In addition, we have

1. for $p=2,4$, or 6 , and for all $T>0$, there exists a constant $C>0$ such that

$$
\mathbb{E} \sup _{0 \leq t \leq T}|u(t)|_{L^{p}}^{p} \leq C \quad \text { and } \quad \mathbb{E} \sup _{0 \leq t \leq T}|v(t)|_{L^{p}}^{p} \leq C .
$$

2. for $p=4$, there exists a constant $C>0$ such that for all $T>0$,

$$
\mathbb{E} \sup _{0 \leq t \leq T}|u(t)|_{H_{p}^{1}}^{2} \leq C \quad \text { and } \quad \mathbb{E} \sup _{0 \leq t \leq T}|v(t)|_{H_{p}^{1}}^{2} \leq C
$$

From Theorem 2.1 and the assumption on the Wiener processes we can prove the existence of a unique solution to the original equation.

Corollary 2.2. If Hypothesis 2.1 and Hypothesis 2.2 are satisfied, then there exists a couple of progressively measurable processes $(u, v)$ solving the system of equations (2.1). In addition, we have

1. for $p=2,4$, or 6 , and for all $T>0$, there exists a constant $C>0$ such that

$$
\mathbb{E} \sup _{0 \leq t \leq T}|u(t)|_{L^{p}}^{p} \leq C \quad \text { and } \quad \mathbb{E} \sup _{0 \leq t \leq T}|v(t)|_{L^{p}}^{p} \leq C .
$$

2. for $p=4$, there exists a constant $C>0$ such that for all $T>0$,

$$
\mathbb{E} \sup _{0 \leq t \leq T}|u(t)|_{H_{p}^{1}}^{2} \leq C \quad \text { and } \quad \mathbb{E} \sup _{0 \leq t \leq T}|v(t)|_{H_{p}^{1}}^{2} \leq C .
$$


Proof of Corollary 2.2: In particular, assuming, for the time being, that the correction term is finite, we get as a new system

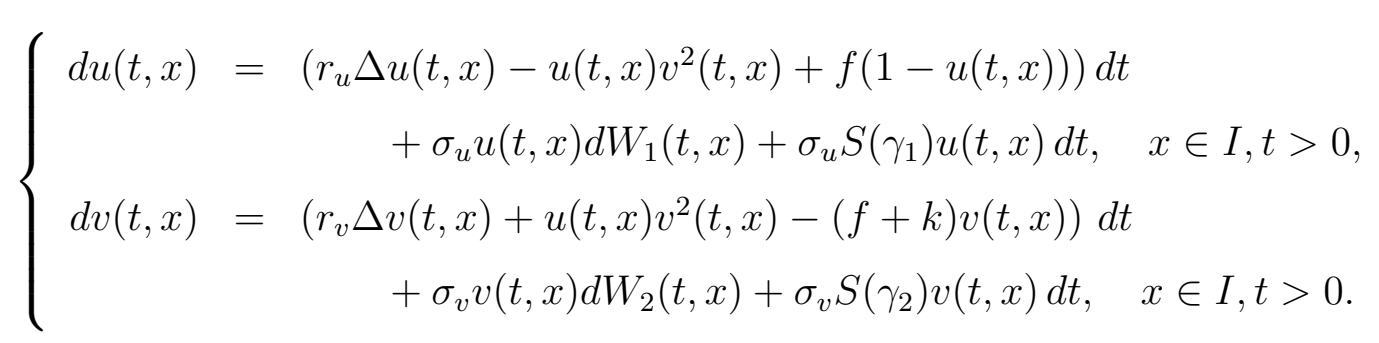

Replacing $f$ by $f-\sigma_{u} S\left(\gamma_{1}\right)$ and $(f+k)$ by $(f+k)-\sigma_{v} S\left(\gamma_{2}\right)$ an application of Theorem 2.1 gives that there is a solution $(u, v)$ to $(2.8)$ both processes being $\mathbb{P}$-a.s. continuous in $H_{2}^{\delta}(I)$ and satisfying (1) and (2). Now, if the process arising by the correction term given by (A.27), i.e.,

$$
\xi_{j}(t)=\frac{1}{2} \int_{0}^{t} \sum_{i, k \in \mathbb{Z}}\left(u(s) \mid \phi_{k}\right)_{\mathcal{H}} \sum_{k \in \mathbb{Z}}\left(\alpha-D \lambda_{k}\right)^{-2 \gamma_{j}} d s
$$

is continuous in $H_{2}^{\delta}(I)$ and satisfies the properties (1) and (2), then we are done. However, this follows by the properties of $u$ and $v$.

Proof. The proof of Theorem 2.1 consists of several steps. First, we show that the system with a truncated nonlinearity can be uniquely solved. In a second step, we show that the solution is a.s. non-negative. In the third step, we give an uniform estimate of $u+v$ in $H_{4}^{1}(I)$. From Sobolev embeddings we get uniform bounds with the $L^{\infty}$-norm. Finally, by these uniform bounds we can globalize the solution in the last step.

Step (i) Fix $m \in \mathbb{N}$. Since we would like to relax the condition on the initial conditions, we first approximate the nonlinear term $u v^{2}$ as follows. Let us define

$$
g_{m}(x):=\left\{\begin{array}{lll}
x & \text { if } & 0<x \leq m \\
\in(m,(m+1)) & \text { if } & m<x<m+1 \\
(m+1) & \text { if } & m+1 \leq x
\end{array}\right.
$$

Between the interval $(m, m+1)$ we interpolate the function by a polynomial function, such that $g_{m}$ is twice continuously differentiable. In particular,

$$
\left.g_{m}^{\prime}(x)\right|_{x=x_{0}}=1, \quad \text { for } \quad x_{0}=m,\left.\quad g_{m}^{\prime}(x)\right|_{x=x_{0}}=0, \quad \text { for } \quad x_{0}=m+1
$$

and

$$
\left.g^{\prime \prime}{ }_{m}(x)\right|_{x=x_{0}}=0, \quad \text { for } \quad x_{0}=m, m+1 .
$$


Let us define the mapping $F_{m}$ by

$$
\begin{aligned}
F_{m}: L^{2}(I) \times L^{4}(I) & \longrightarrow L^{1}(I), \\
(u, v) & \longmapsto F_{m}(u, v) ;
\end{aligned}
$$

by

$$
F_{m}(u, v)(x):=g_{m}(u(x)) g_{m}^{2}(v(x)), \quad x \in I .
$$

The mapping $F_{m}$ is Lipschitz with Lipschitz constant $2(m+1)^{2}$. By Theorem 6.24 [14, p. 178] the following system

$$
\left\{\begin{aligned}
d u_{m}(t, x) & =\left[r_{u} \Delta u_{m}(t, x)-F_{m}\left(u_{m}(t, x), v_{m}(t, x)\right)+\rho+\alpha_{u} u_{m}(t, x)\right] d t+\sigma_{u} u_{m}(t, x) d W_{1}(t, x), \\
u_{m}(0, x) & =u_{0}(x), \quad x \in I,
\end{aligned}\right.
$$

and

$$
\left\{\begin{aligned}
d v_{m}(t, x) & =\left[r_{v} \Delta v_{m}(t, x)+F_{m}\left(u_{m}(t, x), v_{m}(t, x)\right)+\alpha_{v} v_{m}(t, x)\right] d t+\sigma_{v} v_{m}(t, x) d W_{2}(t, x), \\
v_{m}(0, x) & =v_{0}(x), \quad x \in I,
\end{aligned}\right.
$$

has a unique pair of solution $\left\{u_{m}, v_{m}\right\}$, each component belonging to $C\left([0, T] ; L^{2}(I)\right) \cap$ $L^{2}\left([0, T] ; H_{2}^{1}(I)\right)$.

Step (ii) As the next step, we show that each component of the pair of the solution $\left\{u_{m}, v_{m}\right\}$ are non-negative. To show this, we can follow e.g. Theorem 2.3 in [45], or [3, Theorem 2.6.2, p. 42]. Here, we summarize only the idea. In fact it remains to approximate the operator $\Delta$ by, e.g., its Yosida approximation to be able to apply the Itô formula. Let

$$
g_{\delta}(r)=\frac{r^{2}}{\delta+r}, \quad r \in(-\delta, \infty),
$$

and

$$
G_{\delta}(r):=g_{\delta}\left(\left(r^{-}\right)^{2}\right), \quad r \in \mathbb{R} .
$$

Then, $G_{\delta}$ belongs to $C^{2}$ and $G_{\delta}(r)=G_{\delta}^{\prime}(r)=G_{\delta}^{\prime \prime}(r)=0$ for all $r \in[0, \infty),\left|G_{\delta}^{\prime}(r)\right| \leq 2 r^{-}$, and $0 \leq G_{\delta}^{\prime \prime}(r) \leq 8$. Now, define $\phi_{\delta}: L^{2}(I) \rightarrow \mathbb{R}$ by

$$
\phi_{\delta}(w)=\int_{I} G_{\delta}(w(\xi)) d \xi, \quad w \in L^{2}(I) .
$$

Observe, $\phi_{\delta}$ is twice uniformly continuous on bounded subsets, and such that the Itô formula can be applied (see Theorem 4.32 [14, p. 107]. Applying the Itô formula to $\phi_{\delta}\left(u_{m}(t)\right)$ where 
$u_{m}(t)$ solves 2.9$)$, we get

$$
\begin{aligned}
& \mathbb{E} \phi_{\delta}\left(u_{m}(t)\right)+r_{u} \mathbb{E} \int_{0}^{t}\left\langle\Delta u_{m}(s), D \phi_{\delta}\left(u_{m}(s)\right)\right\rangle d s=\phi_{\delta}\left(u_{0}\right)-\mathbb{E} \int_{0}^{t}\left\langle u_{m}(s) v_{m}(s)^{2}, D \phi_{\delta}\left(u_{m}(s)\right)\right\rangle d s \\
& \quad+\alpha_{u} \mathbb{E} \int_{0}^{t}\left\langle u_{m}(s), D \phi_{\delta}\left(u_{m}(s)\right)\right\rangle d s+\frac{\sigma_{u}^{2}}{2} \mathbb{E} \int_{0}^{t} \operatorname{Tr}\left(D^{2} G_{\delta}\left(u_{m}(s)\right)\left[M\left(u_{m}(s)\right) Q^{\frac{1}{2}}\right]\left[M\left(u_{m}(s)\right) Q^{\frac{1}{2}}\right]^{*}\right) d s
\end{aligned}
$$

Note, that

$$
\left\langle\Delta u_{m}(s), D \phi_{\delta}\left(u_{m}(s)\right)\right\rangle=\int_{I}\left(\nabla u_{m}(s, x)\right)^{2} \phi_{\delta}^{\prime \prime}\left(u_{m}(s, x)\right) d x \geq 0 .
$$

Due to A.19, we know

$$
\mathbb{E} \int_{0}^{t} \operatorname{Tr}\left(D^{2} G_{\delta}\left(u_{m}(s)\right)\left[M\left(u_{m}(s)\right)\right]\left[M\left(u_{m}(s)\right)\right]\right) d s \leq 8 \mathbb{E} \int_{0}^{t}\left|u_{m}^{-}(s)\right|_{L^{2}}^{2} d s .
$$

A similar arguments works for $v_{m}$.

$$
\left\langle u_{m}(s) v_{m}(s)^{2}, D \phi_{\delta}\left(u_{m}(s)\right)\right\rangle=\int_{I}\left(u_{m}(s, x)^{-}\right)^{2} v_{m}^{2}(s, x) d x \leq(m+1)^{2} \int_{I}\left|u_{m}(s)^{-}\right|_{L^{2}}^{2},
$$

and

$$
\left\langle u_{m}(s), D \phi_{\delta}\left(u_{m}(s)\right)\right\rangle \leq\left|u_{m}^{-}(s)\right|_{L^{2}}^{2} .
$$

Collecting all together and applying the Grownwall Lemma give $\mathbb{E} \phi_{\delta}\left(u_{m}(t)\right)=0$. Taking the limit $\delta \rightarrow 0$ gives the assertion. Similarly, one can proof that $v_{m}$ is $\mathbb{P}$-a.s. non-negative.

Step (iii) In this step we will show that there exists some bounds on $\mathbb{E}\left|u_{m}\right|_{L^{p}}^{p}$, which are uniform in $m \in \mathbb{N}$.

Claim 2.1. For any even integer $2 \leq p<\infty$ and initial condition satisfying $\mathbb{E}\left|u_{0}\right|_{L^{p}}^{p}, \mathbb{E}\left|v_{0}\right|_{L^{p}}^{p}<$ $\infty$, there exist constants $C_{1}, C_{2}, C_{3}>0$ such that

$$
\mathbb{E} \sup _{0 \leq s \leq T}\left|u_{m}(s)\right|_{L^{p}}^{p} \leq C(T)\left(C_{0}+\mathbb{E}\left|u_{0}\right|_{L^{p}}^{p}\right), \quad \forall m \in \mathbb{N} .
$$

For any even integer $2 \leq p<\infty$, there exist constants $C_{1}, C_{2}, C_{3}>0$ such that

$$
\mathbb{E} \int_{0}^{T} \int_{I} u_{m}^{p-2}(s, x)\left(\nabla u_{m}(s, x)\right)^{2} d x d s \leq C(T)\left(C_{0}+\mathbb{E}\left|u_{0}\right|_{L^{p}}^{p}\right), \quad \forall m \in \mathbb{N} .
$$

Proof. Let us put first $p=2$. The calculations are straight forward using the variational approach. Let us remind that we have equation (2.9) and the definition of the multiplication operator $M$ defined in A.9

$$
d u_{m}(t)=r_{u} A u_{m}(t) d t-F_{m}\left(u_{m}, v_{m}\right)(t)+\alpha_{u} u_{m}(t)+\sigma_{u} M\left(u_{m}(t)\right) d W_{1}(t),
$$


respectively,

$$
d u_{m}(t)=r_{u} A u_{m}(t) d t-F_{m}\left(u_{m}, v_{m}\right)(t)+\alpha_{u} u_{m}(t)+\sum_{k, l \in \mathbb{Z}}\left\langle u_{m}(t), \psi_{l}\right\rangle \psi_{l} h_{k} \beta_{k}(t)
$$

with $h_{k}=\left(\alpha-D \lambda_{k}\right)^{-\frac{\beta}{2}} \psi_{k}$ and $\beta_{k}$ are i.i.d. mutually independent standard Brownian motion. Now, since $\Phi(x)=|x|_{L^{2}}^{2}, D \Phi(x)[h]=\langle x, h\rangle, D^{2} \Phi(x)\left[h^{1}, h^{2}\right]=\left\langle h^{1}, h^{2}\right\rangle$, applying the Itô formula (see Theorem 4.17, [14, p. 105]) to $\Phi(x)=|x|_{L^{2}}^{2}$ and integration by parts give

$$
\begin{aligned}
& d \Phi(u(t))=d \int_{I} u_{m}^{2}(t, x) d x=2 \int_{I} u_{m}(t, x) \Delta u_{m}(t, x) d x d t \\
& \quad-2 \int_{I} u_{m}(t, x) g_{m}\left(u_{m}(t, x)\right) g_{m}^{2}\left(v_{m}(t, x)\right) d x d t+2 \int_{I} u_{m}(t, x)\left(\rho+\alpha_{u} u_{m}(t, x)\right) d x d t \\
& \quad+2 \sum_{k \in \mathbb{Z}}\left\langle u_{m}(t), M\left(u_{m}(t)\right) h_{k}\right\rangle d \beta_{k}(t)+\operatorname{Tr}\left[D^{2} \Phi\left(u_{m}(t)\right)\left[M\left(u_{m}(t)\right) Q^{\frac{1}{2}}\right]\left[M\left(u_{m}(t)\right) Q^{\frac{1}{2}}\right]^{*}\right] d t \\
& =\quad-2 \int_{I}\left(\nabla u_{m}(t, x)\right)^{2} d x d t-2 \int_{I} u_{m}(t, x) g_{m}\left(u_{m}(t, x)\right) g_{m}^{2}\left(v_{m}(t, x)\right) d x d t \\
& \quad+2 \int_{I} u_{m}(t, x)\left(\rho+\alpha_{u} u_{m}(t, x)\right) d x d t \\
& \quad+2 \sum_{k \in \mathbb{Z}}\left\langle u_{m}(t), M\left(u_{m}(t)\right) h_{k}\right\rangle d \beta_{k}(t)+\operatorname{Tr}\left[D^{2} \Phi\left(u_{m}(t)\right)\left[M\left(u_{m}(t)\right) Q^{\frac{1}{2}}\left[M\left(u_{m}(t)\right) Q^{\frac{1}{2}}\right]^{*}\right] d t .\right.
\end{aligned}
$$

Taking the expectation, integrating, and taking into account that the stochastic integral vanishes, we get

$$
\begin{aligned}
& \frac{1}{2} \mathbb{E} \int_{0}^{t} \int_{I} u_{m}^{2}(s, x) d x d s+2 \int_{0}^{t} \int_{I}\left(\nabla u_{m}(s, x)\right)^{2} d x d s \\
& \quad \leq \mathbb{E}\left|u_{0}\right|_{L^{2}}^{2}+2 \int_{0}^{t} \int_{I} u_{m}(s, x) f\left(1-u_{m}(s, x)\right) d x d s+\sigma_{u} \int_{0}^{t} \sum_{k \in \mathbb{Z}}\left|M\left(u_{m}(s)\right) h_{k}\right|_{L^{2}}^{2} d s .
\end{aligned}
$$

By estimate A.19 and Hypothesis 2.1 we have

$$
\int_{0}^{t} \operatorname{Tr}\left[D^{2} \Phi\left(u_{m}(t)\right)\left[M\left(u_{m}(s)\right) Q^{\frac{1}{2}}\left[M\left(u_{m}(s)\right) Q^{\frac{1}{2}}\right]^{*}\right] d s \leq S\left(\gamma_{1}\right) \int_{0}^{t}\left|u_{m}(s)\right|_{L^{2}}^{2} d s,\right.
$$

and therefore, by the Young inequality, we get

$$
\begin{aligned}
& \frac{1}{2} \mathbb{E}\left|u_{m}(t)\right|_{L^{2}}^{2}+2 \int_{0}^{t} \mathbb{E}\left|u_{m}(s)\right|_{H_{2}^{1}}^{2} d s+2 \mathbb{E} \int_{0}^{t} \int_{I} u_{m}(s, x) g_{m}\left(u_{m}(s, x)\right) g_{m}^{2}\left(v_{m}(s, x)\right) d x d s \\
& \quad \leq \mathbb{E}\left|u_{0}\right|_{L^{2}}^{2}+C(\varepsilon)(2 \rho)^{2}+\int_{0}^{t} \mathbb{E}\left|u_{m}(s)\right|_{L^{2}}^{2} d s+\left(\alpha_{u}+C S(\gamma)\right) \int_{0}^{t} \mathbb{E}\left|u_{m}(s)\right|_{L^{2}}^{2} d s .
\end{aligned}
$$


Grownwall's Lemma gives that there exists a constant $C=C(T)>0$ such that

$$
\frac{1}{2} \mathbb{E}\left|u_{m}(t)\right|_{L^{2}}^{2}+2 \int_{0}^{t} \mathbb{E}\left|u_{m}(s)\right|_{H_{2}^{1}}^{2} d s \leq \mathbb{E}\left|u_{0}\right|_{L^{2}}^{2}+C(T), \quad \forall t \in[0, T] .
$$

To estimate the supremum over the time, i.e. $\mathbb{E} \sup _{0 \leq t \leq T}\left|u_{m}(t)\right|_{L^{2}}^{2}$, we have to apply the Burkholder-Davis-Gundy inequality to estimate the stochastic integral

$$
\sum_{k \in \mathbb{Z}}\left\langle u_{m}(t), M\left(u_{m}(t)\right)\left(\alpha-D \lambda_{k}\right)^{-\gamma} h_{k}\right\rangle d \beta_{k}(t) .
$$

Thus, inequality A.19 gives

$\mathbb{E} \sup _{0 \leq s \leq t}\left|\int_{0}^{s} \sum_{k \in \mathbb{Z}}\left\langle u_{m}(r), u_{m}(r) h_{k}\right\rangle d \beta_{k}(r)\right|_{L^{2}} \leq C \mathbb{E}\left(\int_{0}^{t}\left|u_{m}(s)\right|_{L^{2}}^{4} d s\right)^{\frac{1}{2}} \leq C \mathbb{E} \sup _{0 \leq s \leq t}\left|u_{m}(s)\right|_{L^{2}}^{2} t^{\frac{1}{2}}$.

Again, we have by (A.19)

$$
\int_{0}^{t} \operatorname{Tr}\left(D^{2} \Phi\left(u_{m}(s)\right)\left[M\left(u_{m}(s)\right) Q^{\frac{1}{2}}\left[M\left(u_{m}(s)\right) Q^{\frac{1}{2}}\right]^{*}\right) d s \leq S\left(\gamma_{1}\right) \int_{0}^{t}\left|u_{m}(s)\right|_{L^{2}}^{2} d s,\right.
$$

Fix $T^{*}>0$. Integrating up to time $T^{*}$, taking expectation, rearranging, using the Hölder and Young inequality, and taking into account the positivity of $u_{m}(t, x)$, lead to

$$
\begin{aligned}
& \mathbb{E} \sup _{0 \leq t \leq T^{*}} \int_{I} u_{m}^{2}(t, x) d x+2 r_{u} \int_{0}^{T^{*}} \mathbb{E} \int_{I}\left(\nabla u_{m}(t, x)\right)^{2} d x d t \\
& \quad+2 \int_{0}^{T^{*}} \mathbb{E} \int_{I} u_{m}(t, x) g_{m}\left(u_{m}(t, x)\right) g_{m}^{2}\left(u_{m}(t, x)\right) d x d t \\
& \leq \mathbb{E}\left|u_{0}\right|_{L^{2}}^{2}+2 \rho \int_{0}^{T^{*}} \mathbb{E} \int_{I} u_{m}(t, x) d x d t \\
& \quad+2 \alpha_{u} \int_{0}^{T^{*}} \mathbb{E} \int_{I} u_{m}^{2}(t, x) d x d t+2 C_{1}^{Q} \sigma_{u} \mathbb{E} \int_{0}^{T^{*}}\left|u_{m}(t)\right|_{L^{2}}^{2} d t+C \mathbb{E} \sup _{0 \leq s \leq T^{*}}\left|u_{m}(s)\right|_{L^{2}}^{2} T^{* \frac{1}{2}} .
\end{aligned}
$$

Rearranging we get

$$
\begin{aligned}
\mathbb{E} \sup _{0 \leq t \leq T^{*}} \int_{I} u_{m}^{2}(t, x) d x+2 r_{u} \int_{0}^{T^{*}} \mathbb{E} \int_{I}\left(\nabla u_{m}(t, x)\right)^{2} d x d t \\
\quad+2 \int_{0}^{T^{*}} \mathbb{E} \int_{I} u_{m}(t, x) g_{m}\left(u_{m}(t, x)\right) g_{m}^{2}\left(u_{m}(t, x)\right) d x d t \\
\leq \mathbb{E}\left|u_{0}\right|_{L^{2}}^{2}+C(2 \rho)^{2}+\int_{0}^{T^{*}} \mathbb{E}\left|u_{m}(t)\right|_{L^{2}}^{2} d t \\
\quad+2 \alpha_{u} \int_{0}^{T^{*}} \mathbb{E}\left|u_{m}(t)\right|_{L^{2}}^{2} d t+2 C_{1}^{Q} \mathbb{E} \int_{0}^{T^{*}}\left|u_{m}(t)\right|_{L^{2}}^{2} d t+C \mathbb{E} \sup _{0 \leq s \leq T^{*}}\left|u_{m}(s)\right|_{L^{2}}^{2} T^{* \frac{1}{2}}
\end{aligned}
$$


In case $\sqrt{T^{*}} C \leq \frac{1}{2}$, we get by subtracting $\mathbb{E} \sup _{0 \leq s \leq T^{*}}\left|u_{m}(s)\right|_{L^{2}}^{2}$ on both sides

$$
\begin{aligned}
\frac{1}{2} \mathbb{E} \sup _{0 \leq t \leq T^{*}} \int_{I} u_{m}^{2}(t, x) d x+2 r_{u} \int_{0}^{T^{*}} \mathbb{E} \int_{I}\left(\nabla u_{m}(t, x)\right)^{2} d x d t \\
\quad+2 \int_{0}^{T^{*}} \mathbb{E} \int_{I} u_{m}(t, x) g_{m}\left(u_{m}(t, x)\right) g_{m}^{2}\left(u_{m}(t, x)\right) d x d t \\
\leq \mathbb{E}\left|u_{0}\right|_{L^{2}}^{2}+C_{1}+C_{2} \int_{0}^{T^{*}} \mathbb{E}\left|u_{m}(t)\right|_{L^{2}}^{2} d t .
\end{aligned}
$$

Taking into account (2.11) we get

$$
\begin{aligned}
& \frac{1}{2} \mathbb{E} \sup _{0 \leq t \leq T^{*}} \int_{I} u_{m}^{2}(t, x) d x+2 r_{u} \int_{0}^{T^{*}} \mathbb{E} \int_{I}\left(\nabla u_{m}(t, x)\right)^{2} d x d t \\
& \quad+2 \int_{0}^{T^{*}} \mathbb{E} \int_{I} u_{m}(t, x) g_{m}\left(u_{m}(t, x)\right) g_{m}^{2}\left(u_{m}(t, x)\right) d x d t+2 f \int_{0}^{T^{*}} \mathbb{E}\left|u_{m}(t)\right|_{L^{2}}^{2} d t \\
& \leq \mathbb{E}\left|u_{0}\right|_{L^{2}}^{2}+C_{1} \mathbb{E}\left|u_{0}\right|_{L^{2}}^{2}+C_{2}+C\left(T^{*}\right) .
\end{aligned}
$$

Given $T$, we can decompose $[0, T]$ as $\cup_{0 \leq k \leq N-1}\left[k T^{*},(k+1) T^{*}\right]$, and apply inequality (2.14) to each interval $\left[k T^{*},(k+1) T^{*}\right], k=0, \ldots, N-1$. In this way, we extend the estimate to the whole interval $[0, T]$ to prove that the family $\left\{u_{m}: m \in \mathbb{N}\right\}$ can be bounded uniformly for all $m \in \mathbb{N}$ in the supremums norm over time. In particular, we proved the assertion (1) of Theorem 2.1 for the family $\left\{u_{m}: m \in \mathbb{N}\right\}$.

Let $p=4$ and $\Phi(u)=\int_{I} u^{p}(x) d x$. Then $D \Phi(u)[h]=p \int_{I} u^{3}(x) h(x) d x$ and $D^{2} \Phi(u)\left[h^{1}, h^{2}\right]=$ $12 \int_{I} u^{2}(x) h^{1}(x) h^{2}(x) d x$. Recalling that $u_{m}$ is non-negative, we obtain by the Itô formula applied to $\Phi(x)=|x|_{L^{p}}^{p}$

$$
\begin{aligned}
& \Phi(u(T))-\Phi\left(u_{0}\right)=\int_{I} u_{m}^{4}(T, x) d x-\int_{I} u_{m}^{4}(0, x) d x=\int_{0}^{T} \int_{I}\left[r_{u} 4 u_{m}^{3}(t, x) \nabla^{2} u_{m}(t, x)\right. \\
& \left.\quad-4 u_{m}^{3}(t, x) g_{m}\left(u_{m}(t, x)\right) g_{m}^{2}\left(v_{m}(t, x)\right)+4\left(\rho-\alpha_{u} u_{m}(t, x)\right) u_{m}^{3}(t, x)\right] d x d t \\
& \quad+\int_{0}^{T} 4 \sigma_{u} u_{m}^{4}(t, x) d W_{1}(t, x)+\int_{0}^{T} \operatorname{Tr}\left(D^{2} \Phi\left(u_{m}(t)\right)\left[M\left(u_{m}(t)\right) Q^{\frac{1}{2}}\left[M\left(u_{m}(t)\right) Q^{\frac{1}{2}}\right]^{*}\right) d t .\right.
\end{aligned}
$$


Continuing gives

$$
\begin{aligned}
\Phi(u(t))- & \Phi\left(u_{0}\right)+r_{u} 12 \int_{0}^{t} \int_{I} u_{m}^{2}(s, x)\left(\nabla u_{m}(s, x)\right)^{2} d x d s \\
& +p \int_{0}^{t} \int_{I} u_{m}^{3}(t, x) g_{m}\left(u_{m}(t, x)\right) g_{m}^{2}\left(v_{m}(t, x)\right) d x d s \\
= & \Phi(u(T))-\Phi\left(u_{0}\right)+4 \int_{0}^{t} \int_{I}\left(\rho-\alpha_{u} u_{m}(t, x)\right) u_{m}^{3}(s, x) d x d s+p \sigma_{u} \int_{0}^{t} \int_{I} u_{m}^{4}(s, x) d W_{1}(s, x) \\
& +\int_{0}^{t} \operatorname{Tr}\left(D^{2} \Phi\left(u_{m}(s)\right)\left[M\left(u_{m}(s)\right) Q^{\frac{1}{2}}\left[M\left(u_{m}(s)\right) Q^{\frac{1}{2}}\right]^{*}\right) d s .\right.
\end{aligned}
$$

Taking expectation and using integration by parts give

$$
\begin{aligned}
\mathbb{E}\left|u_{m}(t)\right|_{L_{4}}^{4} & +r_{u} 12 \int_{0}^{t} \mathbb{E} \int_{I} u_{m}^{2}(s, x)\left(\nabla u_{m}(s, x)\right)^{2} d x d s \\
& +4 \mathbb{E} \int_{0}^{t} \int_{I} F_{m}\left(u_{m}, v_{m}\right) u_{m}^{3}(s, x) d x d s \leq \mathbb{E}\left|u_{0}\right|_{L_{4}}^{4} \\
& +C_{1} \mathbb{E} \int_{0}^{t} \int_{I} u_{m}^{3}(s, x) d x d s+C_{3} \mathbb{E} \int_{0}^{t}\left|u_{m}(s)\right|_{L^{4}}^{4} d s .
\end{aligned}
$$

We get by some rearrangements and Gronwall's Lemma

$$
\mathbb{E}\left|u_{m}(t)\right|_{L_{4}}^{4}+r_{u} 12 \int_{0}^{t} \mathbb{E} \int_{I} u_{m}^{2}(s, x)\left(\nabla u_{m}(s, x)\right)^{2} d x d s \leq \mathbb{E}\left|u_{0}\right|_{L^{4}}^{4}+C(T) .
$$

To estimate the supremum, we apply again A.21 and get

$$
\mathbb{E} \sup _{0 \leq t \leq T}\left|\int_{0}^{t} \int_{I} u_{m}^{4}(s, x) d W_{1}(s, x)\right| \leq S\left(\gamma_{1}\right) \mathbb{E}\left(\int_{0}^{T}\left|u_{m}^{4}(s)\right|_{L^{2}}^{2} d s\right)^{\frac{1}{2}} .
$$

Applying the Hölder inequality, Sobolev embedding, and then the Young inequality gives for 
July 25, 2019

$\varepsilon, \tilde{\varepsilon}>0$

$$
\begin{aligned}
& \mathbb{E} \sup _{0 \leq t \leq T}\left|\int_{0}^{t} \int_{I} u_{m}^{4}(s, x) d W_{1}(s, x)\right| \leq S\left(\gamma_{1}\right) \mathbb{E}\left(\int_{0}^{T}\left|u_{m}^{2}(s)\right|_{L^{\infty}}^{2}\left|u_{m}^{2}(s)\right|_{L^{2}}^{2} d s\right)^{\frac{1}{2}} \\
& \leq S\left(\gamma_{1}\right) \mathbb{E}\left(\int_{0}^{T}\left|u_{m}^{2}(s)\right|_{H_{2}^{1}}^{2}\left|u_{m}(s)\right|_{L^{4}}^{2} d s\right)^{\frac{1}{2}} \\
& \leq S\left(\gamma_{1}\right) \mathbb{E}\left(\int_{0}^{T}\left(\int_{I} u_{m}^{2}(s, x)\left(\nabla u_{m}(s, x)\right)^{2} d x\right) d s \sup _{0 \leq s \leq T}\left|u_{m}(s)\right|_{L^{4}}^{2}\right)^{\frac{1}{2}} \\
& \leq \varepsilon S\left(\gamma_{1}\right) \mathbb{E} \int_{0}^{T}\left(\int_{I} u_{m}^{2}(s, x)\left(\nabla u_{m}(s, x)\right)^{2} d x\right) d s+C(\varepsilon) \mathbb{E} \sup _{0 \leq s \leq T}\left|u_{m}(s)\right|_{L^{4}}^{2} \\
& \leq \varepsilon S\left(\gamma_{1}\right) \mathbb{E} \int_{0}^{T} \int_{I} u_{m}^{2}(s, x)\left(\nabla u_{m}(s, x)\right)^{2} d x d s+\tilde{\varepsilon} \mathbb{E} \sup _{0 \leq s \leq T}\left|u_{m}(s)\right|_{L^{4}}^{4}+C(\varepsilon, \tilde{\varepsilon}) .
\end{aligned}
$$

Again, the trace is given by

$$
\frac{1}{2} \operatorname{Tr}\left(D^{2} \Phi\left(u_{m}(s)\right)\left[M\left(u_{m}(s)\right) Q^{\frac{1}{2}}\right]\left[M(u(s)) Q^{\frac{1}{2}}\right]^{*}\right)=S(\gamma)\left|u_{m}(s)\right|_{L^{4}}^{4} .
$$

Therefore, taking $\varepsilon$ and $\tilde{\varepsilon}$ sufficiently small

$$
\begin{aligned}
& \frac{1}{2} \mathbb{E} \sup _{0 \leq t \leq T}\left|u_{m}(t)\right|_{L_{4}}^{4}+r_{u} \mathbb{E} \int_{0}^{T} \int_{I} u_{m}^{2}(s, x)\left[\nabla u_{m}(s, x)\right]^{2} d x d s \\
& \quad+4 \mathbb{E} \int_{0}^{T} \int_{I} F_{m}\left(u_{m}, v_{m}\right)(s) u_{m}^{3}(s, x) d x d s \leq \mathbb{E}\left|u_{0}\right|_{L_{4}}^{4} \\
& \quad+4 \mathbb{E} \int_{0}^{T} \int_{I}\left(\rho+\alpha_{u} u_{m}(s, x)\right) u_{m}^{3}(s, x) d x d s+C \mathbb{E} \int_{0}^{T} \int_{I} u_{m}^{4}(s, x) d x d s+C(\varepsilon, \tilde{\varepsilon}) .
\end{aligned}
$$

Due to (2.15) the terms in the RHS are bounded and there exists a constant $C=C(T)>0$ such that

$$
\mathbb{E} \sup _{0 \leq t \leq T}\left|u_{m}(t)\right|_{L_{4}}^{4} \leq C(T) \mathbb{E}\left|u_{0}\right|_{L^{4}}^{4}
$$

Step (iv) Let us define $w_{m}=u_{m}+v_{m}$ and $w_{0}=u_{0}+v_{0}$. Here, we will prove the following claim:

Claim 2.2. Under the Hypothesis 2.9 (ii), the following estimates are valid: 
1. There exists a constant $C=C(T)>0$ such that

$$
\mathbb{E} \sup _{0 \leq t \leq T}\left|w_{m}(t)\right|_{L^{2}}^{2}, \int_{0}^{T} \mathbb{E}\left|\nabla u_{m}(s)\right|_{L^{2}}^{2} d s, \mathbb{E} \int_{0}^{T}\left|\nabla v_{m}(s)\right|_{L^{2}}^{2} d s \leq C, \quad m \in \mathbb{N} .
$$

2. for any even integer $p \geq 2$, there exists a constant $C=C(T, p)>0$ such that

$$
\mathbb{E} \sup _{0 \leq t \leq T}\left|w_{m}(t)\right|_{L^{p}}^{p} \leq C, \quad m \in \mathbb{N}
$$

and

$$
\begin{aligned}
& \mathbb{E} \int_{0}^{t} \int_{I}\left|u_{m}^{k-1}(s, x) v_{m}^{p-1-k}(s, x)\left(\nabla u_{m}(s, x)\right)^{2}\right| d x d s \leq C, \quad k=1 \ldots, p-1, \quad m \in \mathbb{N}, \\
& \mathbb{E} \int_{0}^{t} \int_{I}\left|u_{m}^{k}(s, x) v_{m}^{p-2-k}(s, x)\left(\nabla v_{m}(s, x)\right)^{2}\right| d x d s \leq C, \quad k=0, \ldots, p-2, \quad m \in \mathbb{N} .
\end{aligned}
$$

3. In addition, there exists a constant $C=C(T)>0$ such that

$$
\mathbb{E} \int_{0}^{t} \int_{I}\left|u_{m}^{k-1}(s, x) v_{m}^{p-1-k}(s, x) \nabla u_{m}(s, x) \nabla v_{m}(s, x)\right| d x d s \leq C, \quad k=0, \ldots, p-2, \quad m \in \mathbb{N} .
$$

Proof of Claim 2.2: To show (1) and (2) first note that $w_{m}$ solves

$$
\left\{\begin{aligned}
d w_{m}(t, x)= & \left(r_{u} \Delta u_{m}(t, x)+r_{v} \Delta v_{m}(t, x)+\alpha_{u} w_{m}(t, x)-\left(\alpha_{u}-\alpha_{v}\right) v_{m}(t, x)+\rho\right) d t \\
& +\sigma_{u} u_{m}(t, x) d W_{1}(t, x)+\sigma_{v} v_{m}(t, x) d W_{2}(t, x), \\
w_{m}(0, x)= & u_{0}(x)+v_{0}(x),
\end{aligned}\right.
$$

We denote the inner product in $L^{2}(I)$ by $\langle\cdot, \cdot\rangle$. Now, an application of the Itô formula with 


$$
\begin{aligned}
k=- & \left(\alpha_{u}-\alpha_{v}\right) \text { gives } \\
\mid w_{m}( & t)\left.\right|_{L^{2}} ^{2}+\int_{0}^{t}\left(r_{u}\left|\nabla u_{m}(s)\right|_{L^{2}}^{2}+r_{v}\left|\nabla v_{m}(s)\right|_{L^{2}}^{2}\right) d s \\
& +\int_{0}^{t} \alpha_{u}\left\langle w_{m}(s), w_{m}(s)\right\rangle d s \\
\leq \quad & \left|w_{0}\right|_{L^{2}}^{2}+\int_{0}^{t}\left(r_{u}+r_{v}\right)\left\langle\nabla u_{m}(s), \nabla v_{m}(s)\right\rangle d s+\int_{0}^{t}\left\langle w_{m}(s), \alpha_{u}\right\rangle d s \\
& +k \int_{0}^{t}\left\langle w_{m}(s), v_{m}(s)\right\rangle d s+\int_{0}^{t}\left\langle w_{m}(s), \sigma_{u} u_{m}(s) d W_{1}(s)\right\rangle+\int_{0}^{t}\left\langle w_{m}(s), \sigma_{u} v_{m}(s) d W_{2}(s)\right\rangle \\
& +2 \sigma_{u} \sum_{k \in \mathbb{Z}} \lambda_{k}^{\gamma_{1}}\left\langle w_{m}(t), M\left(u_{m}(t)\right) h_{k}\right\rangle d \beta_{k}^{1}(t)+2 \sigma_{v} \sum_{k \in \mathbb{Z}} \lambda_{k}^{\gamma_{2}}\left\langle w_{m}(t), M\left(v_{m}(t)\right) h_{k}\right\rangle d \beta_{k}^{2}(t) \\
& +\sigma_{u} \int_{0}^{t} \operatorname{Tr}\left(D^{2} \Phi\left(w_{m}(s)\right)\left[M\left(u_{m}(s)\right) Q_{2}^{\frac{1}{2}}\left[M\left(u_{m}(s)\right) Q_{2}^{\frac{1}{2}}\right]^{*}\right) d s\right. \\
& +\sigma_{v} \int_{0}^{t} \operatorname{Tr}\left(D^{2} \Phi\left(w_{m}(s)\right)\left[M\left(v_{m}(s)\right)\left[M\left(v_{m}(s)\right)\right]\right) d s .\right.
\end{aligned}
$$

Since $v_{m}(s) \geq 0$ and $u_{m}(s) \geq 0 \mathbb{P} \times$ Leb-a.e., it follows that $\mathbb{P}$-a.e. $\left\langle w_{m}(s), v_{m}(s)\right\rangle \geq 0$. The Young inequality and taking expectation give

$$
\begin{aligned}
& \mathbb{E}\left|w_{m}(t)\right|_{L^{2}}^{2}+\mathbb{E} \int_{0}^{t}\left(r_{u}\left|\nabla u_{m}(s)\right|_{L^{2}}^{2}+\frac{r_{v}}{4}\left|\nabla v_{m}(s)\right|_{L^{2}}^{2}\right) d s \\
& \quad+\frac{r}{2} \int_{0}^{t} \mathbb{E}\left|w_{m}(s)\right|_{L^{2}}^{2} d s \leq \frac{r_{v}}{2\left(r_{u}+r_{v}\right)} \mathbb{E} \int_{0}^{t}\left|\nabla u_{m}(s)\right|_{L^{2}}^{2} d s+k \int_{0}^{t} \mathbb{E}\left\langle w_{m}(s), v_{m}(s)\right\rangle d s \\
& \quad+C \int_{0}^{t} \mathbb{E}\left|w_{m}(s)\right|_{L^{2}}^{2} d s+C \alpha_{u} t+\mathbb{E}\left|w_{0}\right|_{L^{2}}^{2} .
\end{aligned}
$$

In addition,

$$
\left|\int_{0}^{t} \mathbb{E}\left\langle w_{m}(s), v_{m}(s)\right\rangle d s\right| \leq \int_{0}^{t} \mathbb{E}\left|w_{m}(s)\right|_{L^{2}}^{2} d s+\int_{0}^{t} \mathbb{E}\left|v_{m}(s)\right|_{L^{2}}^{2} d s .
$$

Applying Claim 2.1 and Grownwall's Lemma give

$$
\mathbb{E}\left|w_{m}(t)\right|_{L^{2}}^{2}+\mathbb{E} \int_{0}^{t}\left(r_{u}\left|\nabla u_{m}(s)\right|_{L^{2}}^{2}+\frac{r_{v}}{4}\left|\nabla v_{m}(s)\right|_{L^{2}}^{2}\right) d s \leq C_{1}(T) \mathbb{E}\left|w_{0}\right|_{L^{2}}^{2}+C_{2}(T) .
$$

Note, that we took into account that $\left|u_{m}\right|_{L^{2}},\left|v_{m}\right|_{L^{2}} \leq\left|w_{m}\right|_{L^{2}}$. 
Again, to estimate the supremum, we have to apply the Burkholder-Davis-Gundy inequality A.21 inequality and get

$$
\begin{aligned}
& \mathbb{E} \sup _{0 \leq t \leq T}\left|\int_{0}^{t} \int_{I} w_{m}(s, x) u_{m}(s, x) d W_{1}(s, x)\right| \leq C_{1} \mathbb{E}\left(\int_{0}^{T}\left|w_{m}(s) u_{m}(s)\right|_{L^{2}}^{2} d s\right)^{\frac{1}{2}} \\
& \leq C_{1} \mathbb{E}\left(\int_{0}^{T}\left|w_{m}(s)\right|_{L^{2}}^{2}\left|u_{m}(s)\right|_{L^{\infty}}^{2} d s\right)^{\frac{1}{2}} \leq C_{1} \mathbb{E} \int_{0}^{T}\left|w_{m}(s)\right|_{L^{2}}^{2} d s+\mathbb{E} \sup _{0 \leq s \leq T}\left|u_{m}(s)\right|_{H_{2}^{1}}^{2} .
\end{aligned}
$$

To estimate the supremum in the second stochastic integral, we apply the Burkholder-DavisGundy and the Young inequality, but taking into account that the term containing $v_{m}$ have to be cancelled with the LHS, we obtain

$$
\begin{aligned}
& \mathbb{E} \sup _{0 \leq t \leq T}\left|\int_{0}^{t} \int_{I} w_{m}(s, x) \sigma_{v} v_{m}(s, x) d W_{1}(s, x)\right|_{L^{2}} \\
& \leq \frac{r_{v}}{4} \mathbb{E} \sup _{0 \leq s \leq T}\left|v_{m}(s)\right|_{H_{2}^{1}}^{2}+C(T) C_{2} \mathbb{E} \int_{0}^{T}\left|w_{m}(s)\right|_{L^{2}}^{2} d s
\end{aligned}
$$

The Young inequality and taking expectation give

$$
\begin{aligned}
\mathbb{E} \sup _{0 \leq t \leq T}\left|w_{m}(t)\right|_{L^{2}}^{2}+\mathbb{E} \int_{0}^{T}\left(r_{u}\left|\nabla u_{m}(s)\right|_{L^{2}}^{2}+\frac{r_{v}}{4}\left|\nabla v_{m}(s)\right|_{L^{2}}^{2}\right) d s+k \int_{0}^{T} \mathbb{E}\left\langle w_{m}(s), v_{m}(s)\right\rangle d s \\
\quad+\frac{r}{2} \int_{0}^{T} \mathbb{E}\left|w_{m}(s)\right|_{L^{2}}^{2} d s \leq \frac{r_{v}}{2\left(r_{u}+r_{v}\right)} \mathbb{E} \int_{0}^{T}\left|\nabla u_{m}(s)\right|_{L^{2}}^{2} d s \\
\quad+C_{1} \int_{0}^{T} \mathbb{E}\left|w_{m}(s)\right|_{L^{2}}^{2} d s+C_{2} T+\mathbb{E}\left|w_{0}\right|_{L^{2}}^{2} .
\end{aligned}
$$

Applying Claim 2.1 and the Grownwall Lemma give

$$
\mathbb{E} \sup _{0 \leq t \leq T}\left|w_{m}(t)\right|_{L^{2}}^{2}+\mathbb{E} \int_{0}^{T}\left(r_{u}\left|\nabla u_{m}(s)\right|_{L^{2}}^{2}+\frac{r_{v}}{4}\left|\nabla v_{m}(s)\right|_{L^{2}}^{2}\right) d s \leq C_{1}(T) \mathbb{E}\left|w_{0}\right|_{L^{2}}^{2}+C_{2}(T) .
$$

It remains to show Claim 2.2.(2) and (3). For simplicity, we omit in the following the dependence on $x$ and $t$. To show (ii) observe first, that we have for any $u, v \in H_{2}^{2}(I)$ by integration 
by parts

$$
\begin{aligned}
& \int_{I}(u+v)^{p-1}\left(r_{u} \Delta u+r_{v} \Delta v\right) d x \\
& =\sum_{k=0}^{p-1}\left(\begin{array}{c}
p-1 \\
k
\end{array}\right) \int_{I} u^{k} v^{p-1-k}\left(r_{u} \Delta u+r_{v} \Delta v\right) d x \\
& =-\sum_{k=0}^{p-1}\left(\begin{array}{c}
p-1 \\
k
\end{array}\right) \int_{I} \nabla\left(u^{k} v^{p-1-k}\right)\left(r_{u} \nabla u+r_{v} \nabla v\right) d x .
\end{aligned}
$$

We rewrite the inner part of the sum as follows

$$
\begin{aligned}
& \int_{I} \nabla\left(u^{k} v^{p-1-k}\right)\left(r_{u} \nabla u+r_{v} \nabla v\right) d x \\
& \quad=\int_{I}\left(k u^{k-1} v^{p-1-k} \nabla u+(p-1-k) u^{k} v^{p-2-k} \nabla v\right)\left(r_{u} \nabla u+r_{v} \nabla v\right) d x \\
& =\int_{I}\left(r_{u} k u^{k-1} v^{p-1-k}(\nabla u)^{2}+r_{v}(p-1-k) u^{k} v^{p-2-k}(\nabla v)^{2}\right) d x \\
& +\int_{I}\left(r_{v} k u^{k-1} v^{p-1-k} \nabla u \nabla v+r_{u}(p-1-k) u^{k} v^{p-2-k} \nabla v \nabla u\right) d x .
\end{aligned}
$$

Hence,

$$
\begin{aligned}
\int_{I}(u+v)^{p-1}\left(r_{u} \Delta u+r_{v} \Delta v\right) d x & \\
& +\sum_{k=0}^{p-1}\left(\begin{array}{c}
p-1 \\
k
\end{array}\right) \int_{I}\left(r_{u} k u^{k-1} v^{p-1-k}(\nabla u)^{2}+r_{v}(p-1-k) u^{k} v^{p-2-k}(\nabla v)^{2}\right) d x \\
= & -\sum_{k=0}^{p-1}\left(\begin{array}{c}
p-1 \\
k
\end{array}\right) \int_{I}\left(r_{v} k u^{k-1} v^{p-1-k} \nabla u \nabla v+r_{u}(p-1-k) u^{k} v^{p-2-k} \nabla v \nabla u\right) d x .
\end{aligned}
$$


Renumbering gives

$$
\begin{aligned}
\int_{I}(u+ & v)^{p-1}\left(r_{u} \Delta u+r_{v} \Delta v\right) d x \\
& +\sum_{k=0}^{p-1}\left(\begin{array}{c}
p-1 \\
k
\end{array}\right) \int_{I}\left(r_{u} k u^{k-1} v^{p-1-k}(\nabla u)^{2}+r_{v}(p-1-k) u^{k} v^{p-2-k}(\nabla v)^{2}\right) d x \\
= & -\sum_{k=0}^{p-1}\left(\begin{array}{c}
p-1 \\
k
\end{array}\right) \int_{I} r_{u}(p-1-k) u^{k} v^{p-2-k} \nabla v \nabla u d x \\
& -\sum_{k=0}^{p-2}\left(\begin{array}{c}
p-1 \\
k+1
\end{array}\right) \int_{I}\left(r_{v}(k+1) u^{k} v^{p-2-k} \nabla u \nabla v\right) d x
\end{aligned}
$$

We now estimate the RHS. We get for any $\varepsilon>0$

$$
\begin{aligned}
\int_{I}\left|r_{u} u^{k} v^{p-2-k} \nabla v \nabla u\right| d x & \leq r_{u}\left(\int_{I}\left|u^{k} v^{p-2-k}(\nabla u)^{2}\right| d x\right)^{\frac{1}{2}}\left(\int_{I}\left|u^{k} v^{p-2-k}(\nabla v)^{2}\right| d x\right)^{\frac{1}{2}} \\
& \leq r_{u} C_{\varepsilon} \int_{I}\left|u^{k} v^{p-2-k}(\nabla u)^{2}\right| d x+\varepsilon r_{u} \int_{I}\left|u^{k} v^{p-2-k}(\nabla v)^{2}\right| d x .
\end{aligned}
$$

Taking into account that

$$
\left(\begin{array}{l}
p-1 \\
k+1
\end{array}\right)=\frac{p-1-k}{k+1}\left(\begin{array}{c}
p-1 \\
k
\end{array}\right)
$$

and choosing $\varepsilon=\frac{r_{v}}{4 r_{u}}$ we get

$$
\begin{aligned}
\int_{I}(u+ & v)^{p-1}\left(r_{u} \Delta u+r_{v} \Delta v\right) d x \\
& +\sum_{k=0}^{p-1}\left(\begin{array}{c}
p-1 \\
k
\end{array}\right) \int_{I}\left(r_{u} k u^{k-1} v^{p-1-k}(\nabla u)^{2}+r_{v}(p-1-k) u^{k} v^{p-2-k}(\nabla v)^{2}\right) d x \\
\leq & \sum_{k=0}^{p-1}\left(\begin{array}{c}
p-1 \\
k
\end{array}\right)\left\{r_{v} C_{\gamma} k \int_{I}\left|u^{k-1} v^{p-1-k}(\nabla u)^{2}\right| d x+r_{u} C_{\varepsilon}(p-1-k) \int_{I}\left|u^{k} v^{p-2-k}(\nabla u)^{2}\right| d x\right\} \\
& +\sum_{k=1}^{p-1}\left(\begin{array}{c}
p-1 \\
k
\end{array}\right) \frac{r_{v}}{4}(p-1-k) \int_{I}\left|u^{k-1} v^{p-1-k}(\nabla v)^{2}\right| d x \\
& +\sum_{k=0}^{p-1}\left(\begin{array}{c}
p-1 \\
k
\end{array}\right) \frac{r_{v}}{4}(p-1-k) \int_{I}\left|u^{k} v^{p-2-k}(\nabla v)^{2}\right| d x .
\end{aligned}
$$


Subtracting the last two terms in the inequality, the terms containing $\nabla u$ are remaining on the RHS. In particular, we have

$$
\begin{aligned}
\int_{I}(u+v)^{p-1}\left(r_{u} \Delta u+r_{v} \Delta v\right) d x & \\
& +\sum_{k=0}^{p-1}\left(\begin{array}{c}
p-1 \\
k
\end{array}\right) \int_{I}\left(r_{u} k u^{k-1} v^{p-1-k}(\nabla u)^{2}+\frac{r_{v}}{2}(p-1-k) u^{k} v^{p-2-k}(\nabla v)^{2}\right) d x \\
\leq & \sum_{k=0}^{p-1}\left(\begin{array}{c}
p-1 \\
k
\end{array}\right)\left\{r_{v} C_{\gamma} k \int_{I}\left|u^{k-1} v^{p-1-k}(\nabla u)^{2}\right| d x+r_{u} C_{\varepsilon}(p-1-k) \int_{I}\left|u^{k} v^{p-2-k}(\nabla u)^{2}\right| d x\right\} .
\end{aligned}
$$

Here, applying the Hölder inequality and Young inequality with $q=\frac{p-2}{p-1-k}$ and $q^{\prime}=\frac{p-2}{k-1}$, we get for $\gamma=\frac{1}{q}$

$$
\begin{aligned}
\int_{I}\left|u^{k-1} v^{p-1-k}(\nabla u)^{2}\right| d x & \leq \int_{I}\left|v^{p-1-k}(\nabla u)^{2 \gamma} u^{k-1}(\nabla u)^{2(1-\gamma)}\right| d x \\
\leq \varepsilon \int_{I}\left|v^{p-2}(\nabla u)^{2}\right| d x & +C(\varepsilon) \int_{I}\left|u^{(p-2) \frac{k-1}{k}}(\nabla u)^{2}\right| d x .
\end{aligned}
$$

Again, the first term can be cancelled (by taking $\varepsilon>0$ sufficiently small) with the term

$$
r_{v} v^{p-2}(\nabla v)^{2}
$$

appearing in the sum (for $k=0$ )

$$
\sum_{k=0}^{p-1}\left(r_{u} k u^{k-1} v^{p-1-k}(\nabla u)^{2}+r_{v}(p-1-k) u^{k} v^{p-2-k}(\nabla v)^{2}\right) .
$$

To handle the second term on the RHS we observe

$$
\int_{I}\left|u^{(p-2) \frac{k-1}{k}}(\nabla u)^{2}\right| d x \leq \int_{I}\left|u^{p-2}(\nabla u)^{2}\right| d x+\int_{I}\left|(\nabla u)^{2}\right| d x .
$$

The term on the right hand side can be estimated by Claim 2.1. Going back to problem the 
original problem and applying the Itô formula to $\Phi(u)=\int_{I} u^{p}(x) d x$, we obtain

$$
\begin{aligned}
& \quad\left|w_{m}(t)\right|_{L^{p}}^{p}+\sum_{k=0}^{p-1}\left(\begin{array}{c}
p+1 \\
k
\end{array}\right) \int_{0}^{t} \int_{I}\left(r_{u} k u_{m}^{k-1}(s, x) v_{m}^{p-1-k}(s, x)\left(\nabla u_{m}(s, x)\right)^{2}\right. \\
& \left.\quad+r_{v}(p-1-k) u_{m}^{k}(s, x) v_{m}^{p-2-k}(s, x)\left(\nabla v_{m}(s, x)\right)^{2}\right) d x d s \\
& \leq \quad\left|w_{0}\right|_{L^{p}}^{p}+\sum_{k=0}^{p-1}\left(\begin{array}{c}
p-1 \\
k
\end{array}\right)\left\{r_{v} C_{\gamma} k \int_{0}^{t} \int_{I}\left|u_{m}^{k-1}(s) v_{m}^{p-1-k}(s)\left(\nabla u_{m}(s)\right)^{2}\right| d x\right. \\
& \left.\quad+r_{u} C_{\varepsilon}(p-1-k) \int_{0}^{t} \int_{I}\left|u_{m}^{k}(s) v_{m}^{p-2-k}(s)\left(\nabla u_{m}(s)\right)^{2}\right| d x\right\} \\
& \quad+\int_{I}\left|u_{m}^{p-2}(s)\left(\nabla u_{m}(s)\right)^{2}\right| d x+\int_{I}\left|\left(\nabla u_{m}(s)\right)^{2}\right| d x \\
& +k p \int_{0}^{t} \int_{I} w_{m}^{p-1}(s, x) v_{m}(s, x) d x d s+\alpha_{u} p \int_{0}^{t}\left|w_{m}^{p}(s)\right|_{L^{p}} d s \\
& +p \sigma_{u} \int_{0}^{t} \int_{I} w_{m}^{p-1}(s, x) u_{m}(s, x) d W_{1}(s, x)+\int_{0}^{t} \operatorname{Tr}\left(D^{2} \Phi\left(w_{m}(t)\right) M\left(u_{m}(s)\right) Q^{\frac{1}{2}}\left[M\left(u_{m}(s)\right) Q^{\frac{1}{2}}\right]^{*}\right) d s \\
& +p \sigma_{v} \int_{0}^{t} \int_{I} w_{m}^{p-1}(s, x) u_{m}(s, x) d W_{2}(s, x)+\int_{0}^{t} \operatorname{Tr}\left(D^{2} \Phi\left(w_{m}(t)\right) M\left(v_{m}(s)\right) Q^{\frac{1}{2}}\left[M\left(v_{m}(s)\right) Q^{\frac{1}{2}}\right]^{*}\right) d s
\end{aligned}
$$

Estimating the trace A.19 we obtain

$$
\begin{aligned}
& \operatorname{Tr}\left(D^{2} \Phi\left(w_{m}(t)\right)\left[M\left(u_{m}(s)\right) Q^{\frac{1}{2}}\left[M\left(u_{m}(s)\right) Q^{\frac{1}{2}}\right]^{*}\right)\right. \leq S(\gamma)\left|w_{m}(s)\right|_{L^{p}}^{p}, \\
& \text { and } \operatorname{Tr}\left(D^{2} \Phi\left(w_{m}(t)\right) M\left(v_{m}(s)\right) Q^{\frac{1}{2}}\left[M\left(v_{m}(s)\right) Q^{\frac{1}{2}}\right]^{*}\right) \leq S(\gamma)\left|w_{m}(s)\right|_{L^{p}}^{p}
\end{aligned}
$$

Taking expectation, Gronwall's Lemma, and Claim 2.2, we verify that there exists a $C>0$ such that

$$
\sup _{0 \leq t \leq T} \mathbb{E}\left|w_{m}(t)\right|_{L^{p}}^{p} \leq C
$$

Step (v) In the next step, in order to control the $L^{\infty}$-norm, we will give an estimate of the $H_{p}^{\gamma}(I)$ norm for $\gamma>\frac{d}{p}$. In particular, we will proof the following Claim.

Claim 2.3. There exists a constant $C>0$ such that

1. for $u_{0} \in H_{4}^{1}(I)$ there exists a constant $C>0$ such that

$$
\mathbb{E} \sup _{0 \leq t \leq T} \int_{I}\left|\nabla u_{m}(t, x)\right|^{4} d x \leq C(T)\left(1+\mathbb{E}\left|\nabla u_{0}\right|_{L^{4}}^{4}\right), \quad m \in \mathbb{N} .
$$


2. for $v_{0} \in H_{4}^{1}(I)$ there exists a constant $C=C(T)>0$ such that

$$
\mathbb{E} \sup _{0 \leq t \leq T} \int_{I}\left|\nabla v_{m}(t, x)\right|^{4} d x \leq C(T)\left(1+\mathbb{E}\left|\nabla v_{0}\right|_{L^{4}}^{4}\right), \quad m \in \mathbb{N} .
$$

Proof. Since to show the uniform bounds for $u_{m}$ and $v_{m}$ are quite similar, we will only tackle the proof of the uniform bound for $v_{m}$. Before showing the assertion, we have to show that there exists a constant $C>0$ such that

$$
\mathbb{E} \int_{I}\left|\nabla v_{m}(t, x)\right|^{2} d x \leq C\left(1+\mathbb{E}\left|\nabla v_{0}\right|_{L^{2}}^{2}\right), \quad m \in \mathbb{N}
$$

Here, first, note that by the Itô formula we have $p=2$

$$
\begin{gathered}
\left|\nabla v_{m}(t)\right|_{L^{2}}^{2}+2 \int_{0}^{t} \int_{I}\left(\Delta v_{m}(s, x)\right)^{2} d x d s \leq\left|\nabla v_{0}\right|_{L^{2}}^{2} \\
+\int_{0}^{t} \int_{I} \nabla v_{m}(s, x) \nabla\left(u_{m}(s, x) v_{m}^{2}(s, x)\right) d x d s-2(f+k) \int_{0}^{t} \int_{I}\left(\nabla v_{m}(s, x)\right)^{2} d x d s \\
+\int_{0}^{t} \int_{I} \sigma_{v}\left(\nabla v_{m}(s, x)\right) \nabla v_{m}(s, x) d W_{2}(s, x) \\
+\sigma_{u} \int_{0}^{t} \operatorname{Tr}\left(D \Phi\left(\nabla v_{m}(s)\right)\left[M\left(v_{m}(s)\right) Q^{\frac{1}{2}}\right]\left[M\left(v_{m}(s)\right) Q^{\frac{1}{2}}\right]^{*}\right) d s .
\end{gathered}
$$

The Cauchy-Schwarz and Young inequality give

$$
\begin{aligned}
\int_{I} \Delta v_{m}(s, x) u_{m}(s, x) v_{m}^{2}(s, x) d x & \leq \varepsilon \int_{I}\left(\Delta v_{m}(s, x)\right)^{2} d x+C(\varepsilon) \int_{I} u_{m}^{2}(s, x) v_{m}^{4}(s, x) d x \\
& \leq \varepsilon \int_{I}\left(\Delta v_{m}(s, x)\right)^{2} d x+C(\varepsilon) \int_{I} w_{m}^{6}(s, x) d x
\end{aligned}
$$

Due to Claim 2.2.(ii), the second term is bounded uniformly in $m \in \mathbb{N}$, the first term can be cancelled. Next, we have by the Burkholder-Davis-Gundy inequality

$$
\mathbb{E} \sup _{0 \leq t \leq T}\left|\int_{0}^{t} \int_{I} \sigma_{v}\left(\nabla v_{m}(s, x)\right) \nabla v_{m}(s, x) d W_{2}(s, x)\right| \leq \int_{0}^{t}\left|\left(\nabla v_{m}(s)\right)^{2}\right|_{L^{2}}^{2} d s .
$$

The Hölder inequality, Sobolev embedding, and the Young inequality for convolution give

$$
\cdots \leq \varepsilon \mathbb{E} \sup _{0 \leq s \leq t}\left|\nabla v_{m}(s)\right|_{L^{2}}^{2}+C(\varepsilon) \mathbb{E}\left(\int_{0}^{t}\left|\Delta v_{m}(s)\right|_{L^{2}}^{2} d s\right) .
$$


If $\varepsilon>0$ is chosen sufficiently small, the first term can be cancelled with the left hand side. Finally, we use Hypothesis 2.1 and inequality A.19 to get

$$
\mathbb{E} \int_{0}^{t} \operatorname{Tr}\left(D \Phi\left(\nabla v_{m}(s)\right)\left[M\left(v_{m}(s)\right) Q^{\frac{1}{2}}\right]\left[M\left(v_{m}(s)\right) Q^{\frac{1}{2}}\right]^{*}\right) d s \leq \mathbb{E} \int_{0}^{t}\left|\nabla v_{m}(s)\right|_{L^{2}}^{2} d s
$$

In this way we have shown (2.17).

Let $p=4$ and $\Phi(x)=|x|_{L^{p}}^{p}$. Note, that by the Itô formula we have

$$
\begin{aligned}
& \left|\nabla v_{m}(t)\right|_{L^{p}}^{p}+p(p-1) \int_{0}^{t} \int_{I}\left(\nabla v_{m}\right)^{p-2}(s, x)\left(\Delta v_{m}(s, x)\right)^{2} d x d s \\
& \leq|\nabla v(0)|_{L^{p}}^{p}+\int_{0}^{t} \int_{I}(\nabla v(s, x))^{p-2} \Delta v_{m}(s, x) u_{m}(s, x) v_{m}^{2}(s, x) d x d s \\
& \quad-p(f+k) \int_{0}^{t} \int_{I}\left(\nabla v_{m}(s, x)\right)^{p-1} \nabla v_{m}(s, x) d x d s \\
& \quad+p \int_{0}^{t} \int_{I} \sigma_{v}\left(\nabla v_{m}(s, x)\right)^{p-1} \nabla v_{m}(s, x) d W_{2}(s, x) \\
& \quad+\sigma_{v} \int_{0}^{t} \operatorname{Tr}\left[\Phi\left(v_{m}\right)\left[M\left(v_{m}(s)\right) Q^{\frac{1}{2}}\right]\left[M\left(v_{m}(s)\right) Q^{\frac{1}{2}}\right]^{*} d s .\right.
\end{aligned}
$$

The Cauchy-Schwarz inequality gives

$$
\begin{aligned}
& \int_{I}(\nabla v(s, x))^{p-2} \Delta v_{m}(s, x) u_{m}(s, x) v_{m}(s, x)^{2} d x \\
& \quad \leq\left(\int_{I}\left((\nabla v(s, x))^{p-2} \Delta v_{m}(s, x)\right)^{2} d x\right)^{\frac{1}{2}}\left(\int_{I}\left((\nabla v(s, x))^{p-2} u_{m}^{2}(s, x) v_{m}^{4}(s, x) d x\right)^{\frac{1}{2}} .\right.
\end{aligned}
$$

The Young inequality gives

$$
\begin{aligned}
& \int_{I}(\nabla v(s, x))^{p-2} \Delta v_{m}(s, x) u_{m}(s, x) v_{m}(s, x)^{2} d x \\
& \quad \leq \varepsilon \int_{I}\left[(\nabla v(s, x)]^{p-2} \Delta v_{m}(s, x)\right)^{2} d x+C(\varepsilon) \int_{I}\left((\nabla v(s, x))^{p-2} u_{m}^{2}(s, x) v_{m}^{4}(s, x) d x .\right.
\end{aligned}
$$

In addition, the Burkholder-Davis-Gundy inequality and Hypothesis 2.1 give

$$
\begin{gathered}
\mathbb{E} \sup _{0 \leq t \leq T}\left|\int_{0}^{t} \int_{I} \sigma_{v}\left(\nabla v_{m}(s, x)\right)^{p-1} \nabla v_{m}(s, x) d W_{2}(s, x)\right|^{2} \\
\leq \sigma_{v} S\left(\gamma_{2}\right) \mathbb{E}\left(\int_{0}^{t}\left|\left(\nabla v_{m}(s)\right)^{p-1} \nabla v_{m}(s)\right|_{L^{2}}^{2} d s\right)^{\frac{1}{2}} .
\end{gathered}
$$


The Hölder inequality, Sobolev embedding, and the Young inequality give

$$
\ldots \leq C(\varepsilon) \sigma_{v}^{2} S^{2}\left(\gamma_{2}\right) \mathbb{E}\left(\int_{0}^{t}\left|\nabla v_{m}(s)\right|_{L^{p}}^{p} d s\right)+\varepsilon \sigma_{v}^{2} S^{2}\left(\gamma_{2}\right) \mathbb{E}\left(\sup _{0 \leq s \leq t}\left|\nabla v_{m}(s)\right|_{L^{p}}^{p}\right) .
$$

Taking $\varepsilon$ small enough, the second term can be cancelled with the left hand side of equation (2.19). Finally, estimate A.19 gives

$$
\mathbb{E} \int_{0}^{t} \operatorname{Tr}\left[\Phi\left(v_{m}\right)\left[M\left(v_{m}(s)\right) Q^{\frac{1}{2}}\right]\left[M\left(v_{m}(s)\right) Q^{\frac{1}{2}}\right]^{*} d s \leq S(\gamma) C \mathbb{E} \int_{0}^{t}\left|\nabla v_{m}(s)\right|_{L^{p}}^{p} d s .\right.
$$

Going back to equation (2.19), we obtain

$$
\begin{aligned}
C_{1} \mathbb{E} \sup _{0 \leq s \leq t}\left|\nabla v_{m}(s)\right|_{L^{p}}^{p}+C_{2} p(p-1) \mathbb{E} \int_{0}^{t} \int_{I}\left|\left(\nabla v_{m}\right)^{p-2}(s)\left(\Delta v_{m}(s, x)\right)^{2}\right| d x d s \\
\leq \mathbb{E}\left|\nabla v_{0}\right|_{L^{p}}^{p}+C(\varepsilon) \mathbb{E} \int_{0}^{t} \int_{I}\left(\left(\nabla v_{m}(s, x)\right)^{p-2} u_{m}^{2}(s, x) v_{m}^{4}(s, x) d x d s\right. \\
\quad-p(f+k) \mathbb{E} \int_{0}^{t} \int_{I}\left(\nabla v_{m}(s, x)\right)^{p-1} \nabla v_{m}(s, x) d x d s \\
\quad+\sigma_{v} C(\varepsilon) \mathbb{E} \int_{0}^{t} \int_{I}\left|\left(\nabla v_{m}(s, x)\right)^{p-2} u_{m}^{2}(s, x) v_{m}^{4}(s, x)\right|^{2} d x d s \\
\quad+(C(\varepsilon)+S(\gamma)) \mathbb{E} \int_{0}^{t} \int_{I}\left|\nabla v_{m}(s, x)\right|^{p} d x d s .
\end{aligned}
$$

Observe that the terms

$$
\int_{0}^{t} \int_{I}\left((\nabla v(s, x))^{p-2} u_{m}^{2}(s, x) v_{m}^{4}(s, x) d x d s\right.
$$

and

$$
\mathbb{E} \int_{0}^{t} \int_{I}\left|\left(\nabla v_{m}(s, x)\right)^{p-2} v_{m}^{2}(s, x)\right|^{2} d x d s
$$

can be estimated by Claim 2.2-(iii). Gronwall's Lemma gives the assertion.

Step (vi) In the next step we will define the stopping time depending on the $C_{b}(I)$-norm of the solutions process. However, in order that these stopping times are well defined we have to verify that the solutions processes $\left(u_{m}, v_{m}\right)$ are $\mathbb{P}$-a.s. continuous in $C_{b}(I)$. This is done by showing that $\left(u_{m}, v_{m}\right)$ are $\mathbb{P}$-a.s. continuous in $H_{4}^{\delta}(I)$, where $\delta<1$. Since $d<3$, the continuity in $C_{b}(I)$ follows by embedding Theorems. 
July 25, 2019

Claim 2.4. For any $\delta<1$, there exists a function $C: \mathbb{R}_{0}^{+} \rightarrow \mathbb{R}_{0}^{+}$such that $C(h) \rightarrow 0$ as $h \rightarrow 0$ and

$$
\mathbb{E} \sup _{t \leq s \leq(t+h) \wedge T}\left|u_{m}(s)-u_{m}(t)\right|_{L^{4}}^{4} \leq C(h)\left(1+\mathbb{E}\left|\nabla u_{m}(t)\right|_{L^{4}}^{4}\right), m \in \mathbb{N}, t \in[0, T] .
$$

For any $\delta<1$, there exists a function $C: \mathbb{R}_{0}^{+} \rightarrow \mathbb{R}_{0}^{+}$such that $C(h) \rightarrow 0$ as $h \rightarrow 0$ and

$$
\mathbb{E} \sup _{t \leq s \leq(t+h) \wedge T}\left|v_{m}(s)-v_{m}(t)\right|_{L^{4}}^{4} \leq C(h)\left(1+\mathbb{E}\left|v_{m}(t)\right|_{L^{4}}^{4}\right), m \in \mathbb{N}, t \in[0, T] .
$$

Let us assume by the time being that Claim 2.4 is true. Fist, we have by interpolation of $H_{4}^{\delta}(I)$ for every $s \in[t,(t+h) \wedge T$

$$
\begin{aligned}
\left|u_{m}(s)-u_{m}(t)\right|_{H_{4}^{\delta}} & \leq\left|u_{m}(s)-u_{m}(t)\right|_{L^{4}}^{1-\delta}\left|\nabla u_{m}(s)-\nabla u_{m}(t)\right|_{L^{4}}^{\delta} \\
& \leq\left|u_{m}(s)-u_{m}(t)\right|_{L^{4}}^{1-\delta}\left(\left|\nabla u_{m}(s)\right|_{L^{4}}+\left|\nabla u_{m}(t)\right|_{L^{4}}^{\delta}\right) .
\end{aligned}
$$

In addition, let $q$ and $q^{\prime}$ be integers such that $2 \leq q, q^{\prime}<\infty$ and $\frac{1}{q}+\frac{1}{q^{\prime}}=1$. We take the supremum for every $s \in[t,(t+h) \wedge T$, the expectation, and we use the Hölder inequality on the RHS to get

$$
\begin{aligned}
& \mathbb{E} \sup _{t \leq s \leq(t+h) \wedge T}\left|u_{m}(s)-u_{m}(t)\right|_{H_{4}^{\delta}}^{\gamma} \leq\left(\mathbb{E} \sup _{t \leq s \leq(t+h) \wedge T}\left|u_{m}(s)-u_{m}(t)\right|_{L^{4}}^{\gamma(1-\delta) q}\right)^{\frac{1}{q}} \\
& \quad \times\left(\mathbb{E} \sup _{t \leq s \leq(t+h) \wedge T}\left(\left|\nabla u_{m}(s)\right|_{L^{4}}^{\delta}+\left|\nabla u_{m}(t)\right|_{L^{4}}^{\delta}\right)^{\gamma q^{\prime}}\right)^{\frac{1^{\prime}}{q^{\prime}}} .
\end{aligned}
$$

In the last line we used the identity $(a+b)^{n} \leq C(n)\left(a^{n}+b^{n}\right), n \in \mathbb{N}$. Now we fix $\gamma, q$, and $q^{\prime}$, such that $q(1-\delta) \gamma \leq 4$ and $\gamma q^{\prime} \delta \leq 4$. Under these conditions the RHS can be estimated by Claim 2.4 and Claim 2.3. In particular, if $\gamma=q=q^{\prime}=2$ we have

$$
\mathbb{E} \sup _{t \leq s \leq(t+h) \wedge T}\left|u_{m}(s)-u_{m}(t)\right|_{H_{4}^{\delta}}^{2} \leq C(h)\left(1+\mathbb{E}\left|\nabla u_{m}(t)\right|_{H_{4}^{1}}^{4}\right), m \in \mathbb{N}, t \in[0, T],
$$

and

$$
\mathbb{E} \sup _{t \leq s \leq(t+h) \wedge T}\left|v_{m}(s)-v_{m}(t)\right|_{H_{4}^{\delta}}^{2} \leq C(h)\left(1+\mathbb{E}\left|\nabla v_{m}(t)\right|_{H_{4}^{1}}^{4}\right), m \in \mathbb{N}, t \in[0, T]
$$

Proof. The proof is similar to the proof of the Claim 2.4. Without restriction to the general case we consider the time interval $[0, h \wedge T]$. 
Again an application of the Itô formula to $\Phi(x)=|x|_{L^{4}}^{4}$ gives

$$
\begin{aligned}
\mid v_{m}(t) & -\left.v_{0}\right|_{L^{p}} ^{p}+p(p-2) \int_{0}^{t} \int_{I}\left(v_{m}(s)-v_{0}\right)^{p-2}(s, x)\left(\Delta v_{m}(s, x)-\Delta v(0, x)\right)^{2} d x d s \\
\leq & p(p-2) \int_{0}^{t} \int_{I}\left(v_{m}(s)-v_{0}\right)^{p-2}(s, x)[\Delta v(0, x)]^{2} d x d s \\
& +p \int_{0}^{t} \int_{I}\left(v_{m}(s, x)-v_{0}(0, x)\right)^{p-1}\left[u_{m}(s, x) v_{m}^{2}(s, x)\right] d x d s \\
& -p(f+k) \int_{0}^{t} \int_{I}\left(v_{m}(s, x)-v_{0}(0, x)\right)^{p-1} v_{m}(s, x) d x d s \\
& +p \int_{0}^{t} \int_{I} \sigma_{v}\left(v_{m}(s, x)-v_{0}(0, x)\right)^{p-1} v_{m}(s, x) d W_{2}(s, x) \\
& +\sigma_{v} \int_{0}^{t} \operatorname{Tr}\left(D^{2} \Phi\left(v_{m}(s)-v_{0}(s)\right)\left[M\left(v_{m}(s)\right) Q^{\frac{1}{2}}\right]\left[M\left(v_{m}(s)\right) Q^{\frac{1}{2}}\right]^{*}\right) d s .
\end{aligned}
$$

The Young inequality gives for $p=4$

$$
\left|\int_{0}^{t} \int_{I}\left(v_{m}(t)-v_{0}\right)^{p-2}(s, x)\left[\Delta v_{0}(0, x)\right]^{2} d x d s\right| \leq \int_{0}^{t}\left|v_{m}(s)-v_{0}\right|_{L^{4}}^{4} d s+t\left|\Delta v_{0}\right|_{L^{4}}^{4}
$$

Next,

$$
\begin{aligned}
& \int_{I}\left(v_{m}(s, x)-v_{0}(0, x)\right)^{p-1}\left[u_{m}(s, x) v_{m}^{2}(s, x)\right] d x \\
& \left.\quad \leq C_{1} \mid v_{m}(s)-v_{0}\right]\left.\right|_{L^{p}} ^{p}+C_{2}\left(1+\left|\nabla u_{m}(s)\right|_{L^{2}}^{2}\right)+C_{3}\left(1+\left|v_{m}(s)\right|_{L^{4}}^{4}\right)
\end{aligned}
$$

and

$$
\left|\int_{I}\left(v_{m}(s, x)-v(0, x)\right)^{p-1} v_{m}(s, x) d x\right| \leq\left|v_{m}(s)-v_{0}\right|_{L^{p}}^{p}+\left|v_{m}(s)\right|_{L^{p}}^{p} .
$$

By Claim 2.3 it follows that there exists a $t>0$ such that $\mathbb{E} \int_{0}^{t}\left|\nabla u_{m}(s)\right|_{L^{p}}^{p} d s \leq C \mathbb{E}\left|\nabla u_{0}\right|_{L^{2}}^{2} t$. Besides, by Claim $2.2 \mathbb{E} \int_{0}^{t}\left|v_{m}(s)\right|_{L^{4}}^{4} d s \leq C\left|v_{0}+u_{0}\right|_{L^{4}}^{4} t$. In addition, the Burkholder-DavisGundy inequality and Hypothesis 2.1 give

$$
\begin{aligned}
& \mathbb{E} \sup _{0 \leq t \leq T}\left|\int_{0}^{t} \int_{I} \sigma_{v}\left(v_{m}(s, x)-v_{0}(0, x)\right)^{p-1} v_{m}(s, x) d W_{2}(s, x)\right|^{2} \\
& \leq \sigma_{v} S(\gamma) \mathbb{E}\left(\int_{0}^{t}\left|\left(v_{m}(s)-v_{0}\right)^{p-1} v_{m}(s)\right|_{L^{2}}^{2} d s\right)^{\frac{1}{2}} .
\end{aligned}
$$


By similar calculation as in the step before we get

$$
\ldots \leq \sigma_{v} S(\gamma) \mathbb{E}\left(\int_{0}^{t}\left|v_{m}(s)-v_{0}\right|_{L^{4}}^{2}\left|v_{m}-v_{0}\right|_{L^{4}}^{2}\left|\nabla v_{m}(s)\right|_{L^{2}}^{2} d s\right)^{\frac{1}{2}}
$$

The Young inequality gives

$$
\begin{aligned}
\cdots & \leq \frac{\sqrt{t}}{4} \mathbb{E} \sup _{0 \leq s \leq t}\left|v_{m}(s)-v_{0}\right|_{L^{4}}^{2}+C \sqrt{t}\left(\mathbb{E} \int_{0}^{t}\left|\nabla v_{m}(s)\right|_{L^{2}}^{2} d s\right)^{\frac{1}{2}} \\
& \leq \frac{\sqrt{t}}{4} \mathbb{E}\left(\sup _{0 \leq s \leq T}\left|v_{m}(s)-v_{0}\right|_{L^{4}}^{4}\right)+C t\left(1+\sup _{0 \leq s \leq t} \mathbb{E}\left|\nabla v_{m}(s)\right|_{L^{2}}^{2} .\right.
\end{aligned}
$$

Observe, the first term can be cancelled with the left hand side of equation (2.19). Due to Claim 2.3, the second term is controlled by $C$ t. Finally, we obtain by estimate A.19)

$$
\begin{aligned}
& \int_{0}^{t} \mathbb{E} \operatorname{Tr}\left(D^{2} \Phi\left(v_{m}(s)-v_{0}(s)\right)\left[M\left(v_{m}(s)\right) Q^{\frac{1}{2}}\right]\left[M\left(v_{m}(s)\right) Q^{\frac{1}{2}}\right]^{*}\right) d s \\
& \quad \leq C \int_{0}^{t} \mathbb{E}\left|v_{m}(s, x)-v_{0}(x)\right|^{p-2} v_{m}^{2}(s, x) d x d s .
\end{aligned}
$$

Applying the Hölder inequality gives

$$
\begin{aligned}
& \int_{0}^{t} \mathbb{E} \operatorname{Tr}\left(D^{2} \Phi\left(v_{m}(s)-v_{0}(s)\right)\left[M\left(v_{m}(s)\right) Q^{\frac{1}{2}}\right]\left[M\left(v_{m}(s)\right) Q^{\frac{1}{2}}\right]^{*}\right) d s \\
& \quad \leq C \int_{0}^{t} \mathbb{E}\left|v_{m}(s)-v_{0}\right|_{L^{4}}^{4} d s+\int_{0}^{t} \mathbb{E}\left|v_{m}(s)\right|_{L^{4}}^{4} d s .
\end{aligned}
$$

Collecting all together and analysing term by term, the assertion is shown.

Step (vii) Let $\tau_{m}^{u}:=\left\{t \in(0, T]:\left|u(t)_{m}\right|_{C_{b}} \geq m\right\}, \tau_{m}^{v}:=\left\{t \in(0, T]:\left|v(t)_{m}\right|_{C_{b}} \geq m\right\}$ and $\tau_{m}:=\min \left(\tau_{m}^{u}, \tau_{m}^{v}\right)$. In this step we will show that for $m \rightarrow \infty$ we have $\mathbb{P}\left(\tau_{m}<T\right) \rightarrow 0$. Observe, that for $\delta<1$ the trajectories $[0, T] \ni t \mapsto(u(t), v(t)) \in H_{4}^{\delta}(I) \times H_{4}^{\delta}(I)$ are continuous. Besides, due to the fact that $H_{4}^{\delta}(I) \hookrightarrow C_{b}(I)$ for $\frac{1}{2}<\delta<1$, the trajectories $[0, T] \ni t \mapsto(u(t), v(t)) \in C_{b}(I) \times C_{b}(I)$ are continuous and the stopping times are well defined. In addition, the estimate on $u_{m}$ and $v_{m}$ in Claim 2.3 were independent of $m$. Hence, for all $\delta \in \mathbb{R}$ with $\frac{1}{2}<\delta<1$ there exists a constant $C>0$ such that

$$
\mathbb{E} \sup _{0 \leq t \leq T}\left|u_{m}(t)\right|_{H_{4}^{\delta}}^{4}, \mathbb{E} \sup _{0 \leq t \leq T}\left|v_{m}(t)\right|_{H_{4}^{\delta}}^{p} \leq C, \quad m \in \mathbb{N} .
$$

Due to the embedding $H_{p}^{\delta}(I) \hookrightarrow C_{b}(I)$, there exists a constant $C>0$ such that

$$
\mathbb{E} \sup _{0 \leq t \leq T}\left|u_{m}(t)\right|_{C_{b}}^{p}, \mathbb{E} \sup _{0 \leq t \leq T}\left|v_{m}(t)\right|_{C_{b}}^{p} \leq C, \quad m \in \mathbb{N}
$$


Let us define the stopping time

$$
\tau_{m}^{u}:=\inf _{t \geq 0}\left\{\left|u_{m}\right|_{H_{4}^{\delta}} \geq m\right\} \quad \text { and } \quad \tau_{m}^{v}:=\inf _{t \geq 0}\left\{\left|v_{m}\right|_{H_{4}^{\delta}} \geq m\right\} .
$$

By the definition of $g_{m}$ it follows that for $s \leq \tau_{m}:=\min \left(\tau_{m}^{u}, \tau_{m}^{v}\right)$ we get

$$
F_{m}(u(s), v(s))=F_{m+1}(u(s), v(s))=F(u(s), v(s))
$$

where $F: L^{2}(I) \times L^{\infty}(I) \rightarrow L^{2}(I)$ is the Nemityski operator defined by

$$
F(u, v)(x):=f(u(x), v(x))=u(x) v^{2}(x) .
$$

Hence, on $\left[0, \tau_{m}\right)$ the processes $\left(u_{m}, v_{m}\right)$ and $\left(u_{m+1}, v_{m+1}\right)$ are identical and $\tau_{m} \leq \tau_{m+1}$, for all $m \in \mathbb{N}$. Fix $m \in \mathbb{N}$ and put

$$
A_{m}:=\left\{\omega \in \Omega:|u(s)|_{C_{b}} \leq m \text { and }|v(s)|_{C_{b}} \leq m\right\} .
$$

It is straightforward that there exists a progressively measurable process $\left(u_{m}, v_{m}\right)$ over $\mathfrak{A}=$ $\left(\Omega, \mathcal{F},\left(\mathcal{F}_{t}\right)_{t \in[0, T]}, \mathbb{P}\right)$ such that $\left(u_{m}, v_{m}\right)$ solves $\mathbb{P}$-a.s. the integral equation given by (2.8) up to time $\tau_{m}$. In particular, we have for the conditioned probability

$$
\mathbb{P}\left(\{\text { a solution } u \text { to } 2.8 \text { exists }\} \mid A_{m}\right)=1 \text {. }
$$

Hence, for any $m \in \mathbb{N}$ we can glue together the solution to one process $(u, v)$ with

$$
u(t)=u_{m}(t) \text { and } v(t)=v_{m}(t) \text { when } t \in\left[\tau_{m-1} \wedge T, \tau_{m} \wedge T\right) .
$$

Then, it is straightforward to verify, that

$$
\begin{aligned}
& \mathbb{P}(\{\text { there exists solution to } 2.8\}) \\
& \quad=\lim _{m \rightarrow \infty} \mathbb{P}\left(\{\text { a solution } u \text { to } 2.8 \text { exists }\} \mid A_{m}\right) \mathbb{P}\left(A_{m}\right) .
\end{aligned}
$$

Since $\mathbb{P}\left(\{\right.$ a solution $u$ to 2.8 exists $\left.\} \mid A_{m}\right)=1$, it remains to show that $\lim _{m \rightarrow \infty} \mathbb{P}\left(A_{m}\right)=1$. Then, as $A_{m} \supset A_{m+1}$, it follows automatically that

$$
\mathbb{P}(\{\text { there exists solution to } 2.8\})=1 \text {. }
$$

However, since there exists a constant $C(T)>0$ such that

$$
\mathbb{E} \sup _{0 \leq t \leq T}\left|u_{m}(t)\right|_{C_{b}}^{4}, \mathbb{E} \sup _{0 \leq t \leq T}\left|u_{m}(t)\right|_{C_{b}}^{4} \leq C(T), \quad m \in \mathbb{N},
$$

and, hence,

$$
\mathbb{P}\left(\Omega \backslash A_{m}\right) \leq \frac{C(T)}{m^{4}} \rightarrow 0 .
$$

The solution process is well defined on $A=\lim _{m \rightarrow \infty} A_{m}$, where $\mathbb{P}(A)=1$. In addition, by Claim 2.2 and the non-negativity of the solution item (i), (ii), and (iii) follow. 


\section{Numerical simulations}

The approach of operator splitting may lead to favourable discretisations for various classes of deterministic evolution equations, see HAIRER, WANNER [22] and references given therein. We mention the works [46, 47], which illustrate the use of operator splitting methods in the context of nonlinear Schrödinger equations and confirm that time-adaptivity enhances reliability and efficiency of the numerical simulations; in [47], it is also demonstrated that Fourier spectral space approximations, although constrained to uniform meshes, are superior to locally adaptive finite element space discretisations, due to the retained spectral convergence rate and the applicability of fast Fourier transform techniques. We expect similar conclusions to hold for reaction-diffusion equations with pattern formation, requiring, as well, high resolution in space and time, and hence favour the Fourier spectral method over the finite difference and finite element methods; we point out once again that the simple structure of the space domain and the imposed periodic boundary conditions permit solution representations by Fourier series expansion. Schemes similar to this were considered for the deterministic case in HochBruck, Ostermann [23]. In the context of stochastic evolution equations, operator splitting methods have been studied in BARBU, Röckner [4], Bauzet et al. [5], Bauzet [6], Bessaih et al. [7], BrÉhier, Goudenége [8], Carelli et al. [11], Sango [40], and Karlsen, Storrøsten [24].

For our purposes, it is convenient to cast the stochastic Gray-Scott equations in Itô or Stratonovich formulation, respectively, into the form of an evolutionary system

$$
\begin{gathered}
A_{u}=r_{u} \Delta-\widetilde{\alpha}_{u}, \quad A_{v}=r_{v} \Delta-\widetilde{\alpha}_{v}, \quad g(u, v)=u v^{2}, \\
\left\{\begin{array}{l}
\mathrm{d} u(t)=\left(A_{u} u(t)+\alpha_{u}-g(u(t), v(t))\right) \mathrm{d} t+\sigma_{u} u(t) \mathrm{d} W_{u}(t), \\
\mathrm{d} v(t)=\left(A_{v} v(t)+g(u(t), v(t))\right) \mathrm{d} t+\sigma_{v} v(t) \mathrm{d} W_{v}(t), \\
u(0)=u_{0}, \quad v(0)=v_{0}, \quad t \in(0, T) ;
\end{array}\right.
\end{gathered}
$$

the choice $\widetilde{\alpha}_{u}=\alpha_{u}, \widetilde{\alpha}_{v}=\alpha_{v}$ corresponds to (2.1), and the modification

$$
\widetilde{\alpha}_{u}=\alpha_{u}-\sigma_{u} S(\gamma), \quad \widetilde{\alpha}_{v}=\alpha_{v}-\sigma_{v} S(\gamma), \quad S(\gamma)=\sum_{k \in \mathbb{Z}^{d}}\left(\alpha-D \lambda_{k}\right)^{-2 \gamma}
$$


to (2.6). Moreover, we set

$$
\begin{gathered}
U(t)=\left(\begin{array}{l}
u(t) \\
v(t)
\end{array}\right), \quad U_{0}=\left(\begin{array}{l}
u_{0} \\
v_{0}
\end{array}\right), \\
A_{U}=\left(\begin{array}{cc}
A_{u} & 0 \\
0 & A_{v}
\end{array}\right), \quad G(U(t))=\left(\begin{array}{c}
\alpha_{u}-g(u(t), v(t)) \\
g(u(t), v(t))
\end{array}\right), \\
\Sigma(U(t))=\left(\begin{array}{cc}
\sigma_{u} u(t) & 0 \\
0 & \sigma_{v} v(t)
\end{array}\right), \quad W_{U}(t)=\left(\begin{array}{l}
W_{u}(t) \\
W_{v}(t)
\end{array}\right), \quad t \in[0, T], \\
\left\{\begin{array}{l}
\mathrm{d} U(t)=\left(A_{U} U(t)+G(U(t))\right) \mathrm{d} t+\Sigma(U(t)) \mathrm{d} W_{U}(t), \quad t \in(0, T), \\
U(0)=U_{0} .
\end{array}\right.
\end{gathered}
$$

Deterministic Gray-Scott equations. Let us first discuss the deterministic formulation. A natural approach for the numerical solution of the deterministic Gray-Scott equations or, more generally, of a deterministic evolution equation of the form

$$
\left\{\begin{array}{l}
U^{\prime}(t)=F(U(t))=F_{1}(U(t))+F_{2}(U(t)), \quad t \in(0, T), \\
U(0)=U_{0},
\end{array}\right.
$$

is based on operator splitting, i.e. the defining operator is decomposed into two parts; for each subinterval, defined by a suitably chosen time stepsize $h_{n}>0$, the associated subproblems are solved separately, potentially with specific numerical solvers. More specifically, for the LieTrotter splitting method of classical order one, the linear subproblem

$$
\left\{\begin{array}{l}
V_{1}^{\prime}(t)=F_{1}\left(V_{1}(t)\right), \quad t \in\left(t_{n}, t_{n}+h_{n}\right), \\
V_{1}\left(t_{n}\right)=U_{n} \approx U\left(t_{n}\right),
\end{array}\right.
$$

is resolved; starting from the resulting approximation $V_{1}\left(t_{n}+h_{n}\right)$, the resolution of the nonlinear subproblem

$$
\left\{\begin{array}{l}
V_{2}^{\prime}(t)=F_{2}\left(V_{2}(t)\right), \quad t \in\left(t_{n}, t_{n}+h_{n}\right), \\
V_{2}\left(t_{n}\right)=V_{1}\left(t_{n}+h_{n}\right),
\end{array}\right.
$$

then yields an approximation to the exact solution value

$$
U_{n+1}=V_{2}\left(t_{n}+h_{n}\right) \approx U\left(t_{n}+h_{n}\right) .
$$

The compact formulation of the deterministic Gray-Scott equations makes the natural decomposition of the right-hand side into two parts evident, see (3.2). Let $h_{n}$ be the time-step size. The solution of the linear subproblem

$$
V_{1}^{\prime}(t)=A_{U} V_{1}(t), \quad t \in\left(t_{n}, t_{n}+h_{n}\right),
$$


comprising two decoupled diffusion equations, is formally given by

$$
V_{1}\left(t_{n}+h_{n}\right)=\mathrm{e}^{h A_{U}} V_{1}\left(t_{n}\right) ;
$$

for each component, an explicit solution representation based on a Fourier series expansion is available. Our numerical approximation relies on a truncation of the infinite series and an application of the trapezoidal rule on an equidistant space grid; we use the fast Fourier transform and its inverse for an efficient implementation. Similar techniques in the stochastic setting has been used e.g. by LORD AND Rougemont [32].

For the numerical solution of the second subproblem

$$
V_{2}^{\prime}(t)=G\left(V_{2}(t)\right), \quad t \in\left(t_{n}, t_{n}+h_{n}\right),
$$

comprising the nonlinear reaction terms, we employ a standard explicit solver. More precisely, we retain the equidistant space grid used for the discretisation of the linear subproblem; pointwise evaluation at each grid point yields a system of ordinary differential equations, which we resolve by an explicit Runge-Kutta method.

Stochastic Gray-Scott equations. For the stochastic Gray-Scott equations, we propose to employ the following natural modification of the Lie-Trotter splitting method. We consider instead the linear subproblem

$$
\mathrm{d} V_{1}(t)=A_{U} V_{1}(t) \mathrm{d} t+\Sigma\left(V_{1}(t)\right) \mathrm{d} W_{U}(t), \quad t \in\left(t_{n}, t_{n}+h_{n}\right) ;
$$

in view of the formal representation for the mild solution

$$
V_{1}\left(t_{n}+h_{n}\right)=\mathrm{e}^{h_{n} A_{U}} V_{1}\left(t_{n}\right)+\int_{0}^{h_{n}} \mathrm{e}^{\left(h_{n}-s\right) A_{U}} \Sigma\left(V_{1}\left(t_{n}+s\right)\right) \mathrm{d} W_{U}\left(t_{n}+s\right),
$$

we employ the approximation

$$
V_{1}\left(t_{n}+h_{n}\right) \approx \mathrm{e}^{h_{n} A_{U}}\left(V_{1}\left(t_{n}\right)+\Sigma\left(V_{1}\left(t_{n}\right)\right)\left(W_{U}\left(t_{n}+h_{n}\right)-W_{U}\left(t_{n}\right)\right)\right) .
$$

For the realisation of the increment $W_{U}\left(t_{n}+h_{n}\right)-W_{U}\left(t_{n}\right)$, we generate normally distributed numbers and apply the inverse Laplacian $(1-\Delta)^{-\gamma}$; this, as well as the action of the evolution operator $\mathrm{e}^{h_{n} A_{U}}$, is implemented by fast Fourier transforms. The modification of the nonlinear subproblem is straightforward

$$
\mathrm{d} V_{2}(t)=G\left(V_{2}(t)\right) \mathrm{d} t, \quad t \in\left(t_{n}, t_{n}+h_{n}\right) ;
$$

for its approximate solution, we again apply an explicit solver.

Due to the fact that the solution is a stochastic process, avoidance of blow-up by a suitable reduction of the time stepsizes is slightly more delicate compared to the deterministic GrayScott equations. Even though the probability for such cases is low, large realisations of $W_{U}$ 
may lead to large values. To prevent failure of the code, we follow the simple strategy which we point out shortly in the following paragraph. Fix $t_{n}$. Since we are evaluating pointwise at each grid point $x_{m}, m=\left(m_{1}, m_{2}\right)$, the nonlinearity given by $g(u, v)=u v^{2}$, we can associate to each grid point a couple process $\left(\hat{u}_{m}, \hat{v}_{m}\right)$ following the two coupled differential equations:

$$
\left\{\begin{array}{l}
\dot{\hat{u}}_{m}(t)=-\hat{u}_{m}(t) \hat{v}_{m}^{2}(t)+\alpha_{U}, \\
\dot{\hat{v}}_{m}(t)=\hat{u}_{m}(t) \hat{v}_{m}^{2}(t),
\end{array}\right.
$$

with initial condition $\hat{u}_{m}(0):=\hat{u}_{m}$ and $\hat{v}_{m}(0):=\hat{v}_{m}$, where $\hat{U}_{m}=(\hat{u}, \hat{v})$ is the approximation of $U\left(t_{n}\right)$ gained in the step before. To prevent these ODEs from blowing up in a finite time, we fix our step size by

$$
h_{n} \leq h_{0} \max _{\substack{m=\left(m_{1}, m_{2}\right) \\ \text { is a grid point }}} \hat{u}_{m}(0) \hat{v}_{m}(0)
$$

where, again, $\hat{U}_{n}=(\hat{u}, \hat{v})$ and $\hat{U}_{n}$ is the approximation of $U$. To motivate this choice, let us focus on the second component solving the nonlinear ODE and fix a constant $\eta_{0}>0$. The first component will not be a problem since the nonlinear term has a negative sign and the positive term is a constant. Therefore there will be no blow up in finite time for the first component. Let us consider the solution $\xi=\{\xi(t): t \geq 0\}$ where $\xi$ solves, for a given initial condition $\xi_{0}>0$, the following nonlinear ODE

$$
\dot{\xi}(t)=\eta_{0} \xi^{2}(t), \quad t \geq 0, \quad \xi(0)=\xi_{0} .
$$

The solution $\xi$ solving this ODE is given by

$$
\xi(t)=\frac{\xi_{0}}{1-\xi_{0} \eta_{0} t}, \quad 0 \leq t<\frac{1}{\xi_{0} \eta_{0}} .
$$

In particular, if $t \uparrow \frac{1}{\xi_{0} \eta_{0}}$ then $\xi(t) \rightarrow \infty$. By the choice of 3.3 , we know that

$$
1+h_{0}=\frac{1}{1-\eta_{0} \xi_{0} h_{n}}
$$

and we get a time-step $h_{n}$, such that the solution $\xi$ at time $t=h_{n}$ with constant $\eta_{0}$ will not exceed $\left(1+h_{0}\right) \xi_{0}$. In this way, we get our condition for the choice of the adapted time step and we prevent the failure of the time integration due to large realisations of the Wiener processes.

Numerical results. In Figure 1, we display the initial states prescribed for the twodimensional deterministic and stochastic Gray-Scott equations (3.1)-(3.2). For two exponents $\gamma>0$, the effect of the inverse Laplacian $(1-\Delta)^{-\gamma}$ on a set of normally distributed numbers is illustrated in Figure 2, Realisations of the numerical solution processes are shown in Figure 3 for 

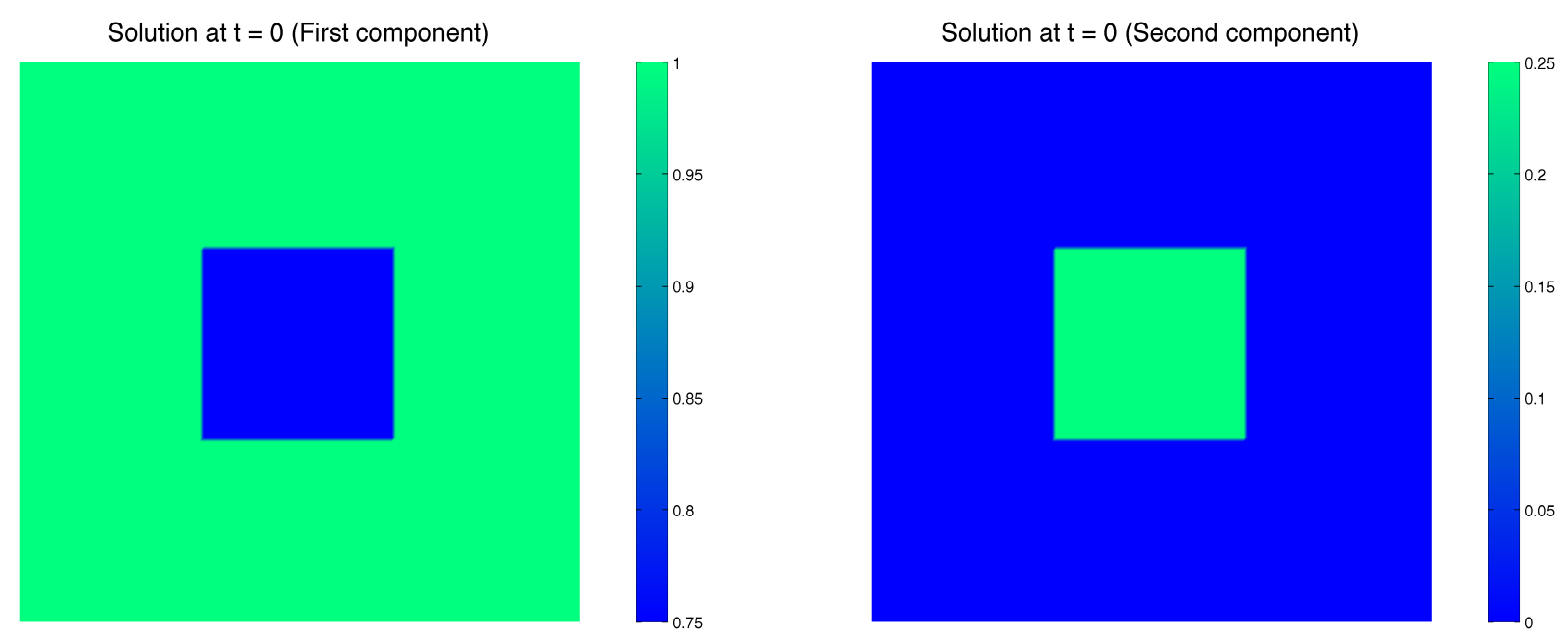

Figure 1: Deterministic and stochastic Gray-Scott equations. Prescribed initial states.

a certain choice of the parameters $r_{u}, r_{v}, \widetilde{\alpha}_{u}, \widetilde{\alpha}_{v}, \sigma_{u}, \sigma_{v}, \gamma$. The first row corresponds to the first solution component and the deterministic case, the second row to the Stratonovich formulation

$$
\widetilde{\alpha}_{u}=\alpha_{u}-\sigma_{u} S(\gamma), \quad \widetilde{\alpha}_{v}=\alpha_{v}-\sigma_{v} S(\gamma), \quad S(\gamma)=\sum_{k \in \mathbb{Z}^{d}}\left(\alpha-D \lambda_{k}\right)^{-2 \gamma}, \quad \alpha=5, \quad D=1
$$

and the third row to the Itô formulation. In the captions of the figures, we provide links to movies that visualise the creation of patterns and their variation under the influence of stochastic noise.

\section{Appendix A. The stochastic integral, the multiplication operator and some in- equalities}

Provided that a stochastic process $(Y(t))_{t \in[0, T]}$ with values in the space of Hilbert-Schmidt operators from $\mathcal{H}$ to another Hilbert space $\widetilde{\mathcal{K}}$ is progressively measurable on the underlying probability space and fulfills a certain integrability condition

$$
Y: \Omega \times[0, T] \longrightarrow L_{\mathrm{HS}}(\mathcal{H}, \widetilde{\mathcal{K}}), \quad \mathbb{E}\|Y\|_{L^{2}\left([0, T], L_{\mathrm{HS}}(\mathcal{H}, \widetilde{\mathcal{K}})\right)}^{2}<\infty
$$

the stochastic integral, denoted by

$$
J: \Omega \times[0, T] \longrightarrow \widetilde{\mathcal{K}}:(\omega, t) \longmapsto \int_{0}^{t} Y(\omega, s) \mathrm{d} W(\omega, s),
$$




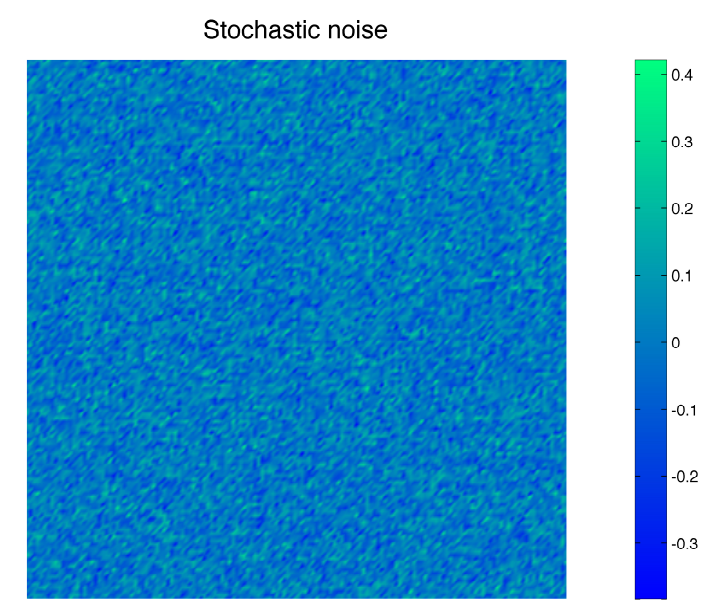

Regularisation by inverse Laplacian $($ Exponent $=0.5)$

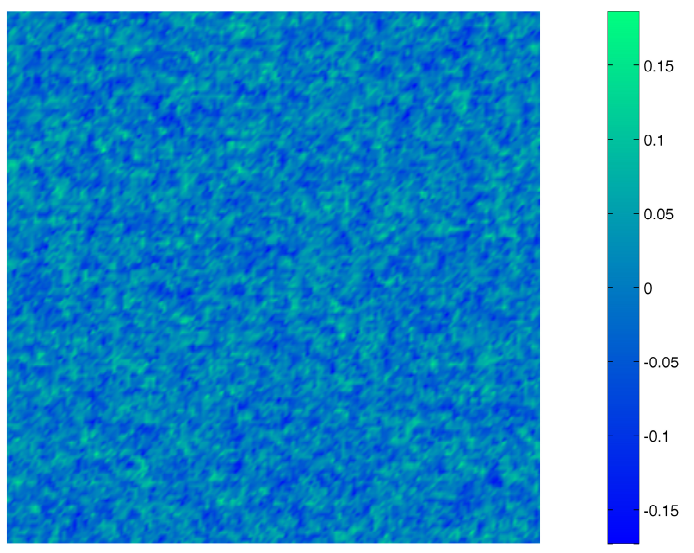

Regularisation by inverse Laplacian (Exponent $=2)$
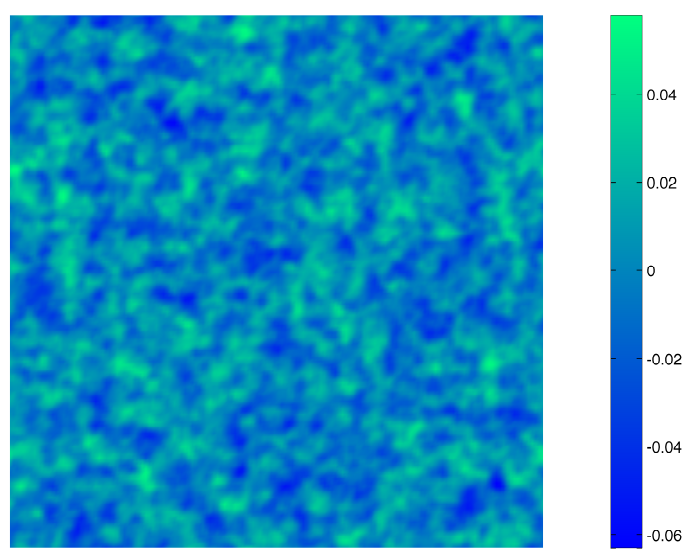

Stochastic noise
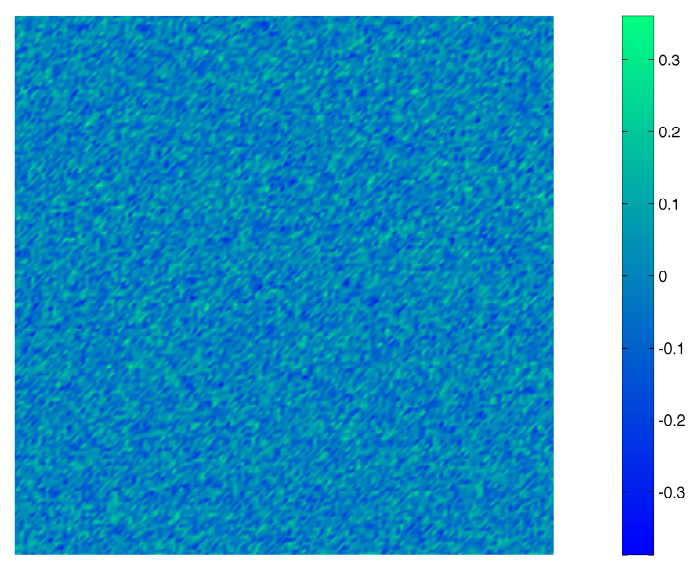

Regularisation by inverse Laplacian $($ Exponent $=0.5)$

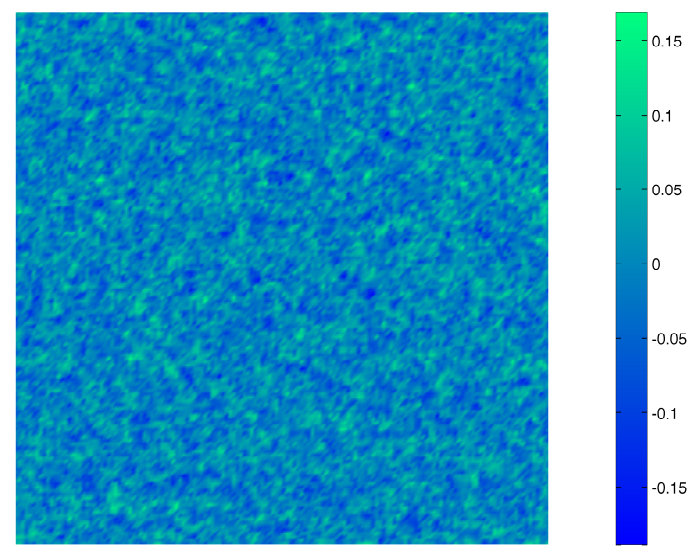

Regularisation by inverse Laplacian (Exponent $=2)$

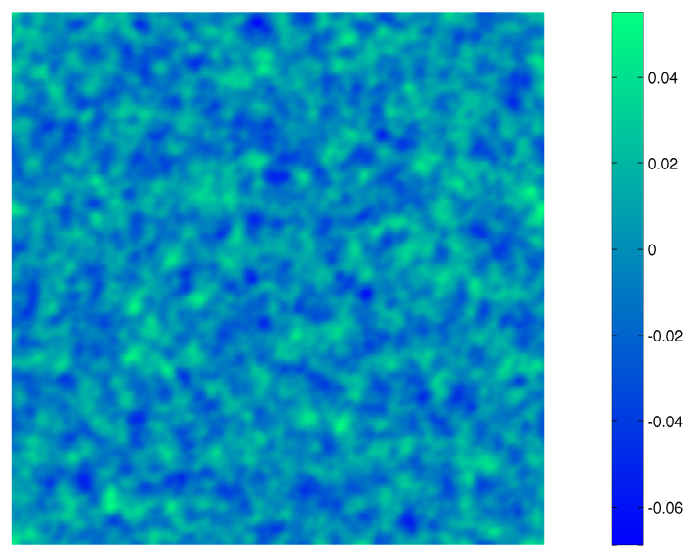

Figure 2: Two realisations of stochastic noise and regularisations by powers of inverse Laplacian $(1-\Delta)^{-\gamma}$, $\gamma \in\{0.5,2\}$. 
Solution at $\mathrm{t}=1500$ (First component) Parameters $(0.16,0.08,0.029,0.086)$, Noise $(0,0,0)$
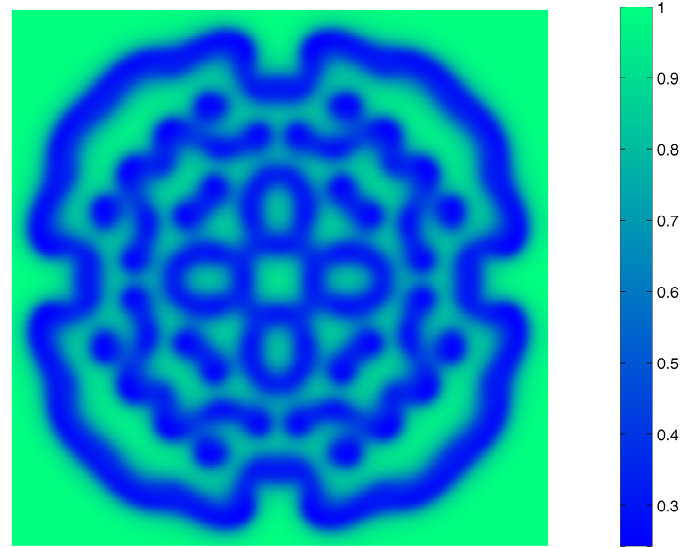

Solution at $\mathrm{t}=1500$ (First component) Parameters $(0.16,0.08,0.024349,0.081349)$, Noise $(0.001,0.001,2)$
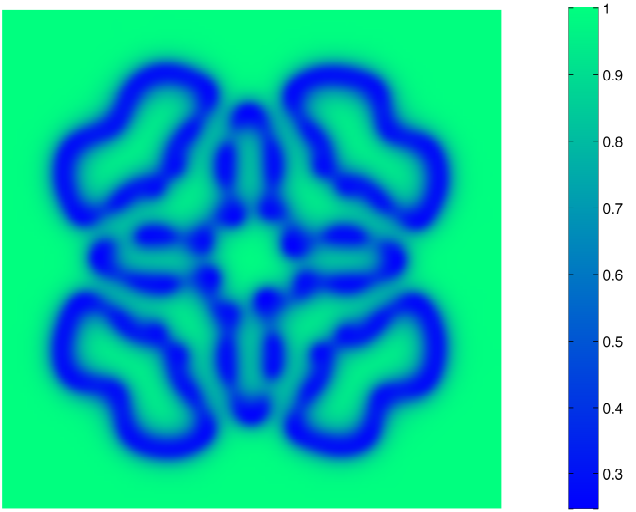

Solution at $\mathrm{t}=1500$ (First component) Parameters $(0.16,0.08,0.029,0.086)$, Noise $(0.001,0.001,2)$

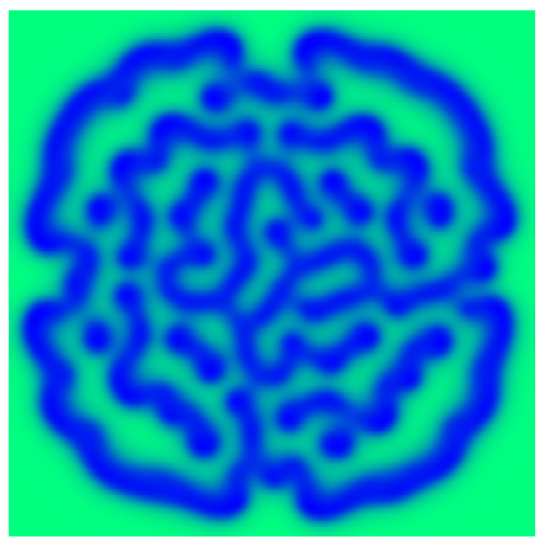

Solution at $\mathrm{t}=3000$ (First component) Parameters $(0.16,0.08,0.029,0.086)$, Noise $(0,0,0)$

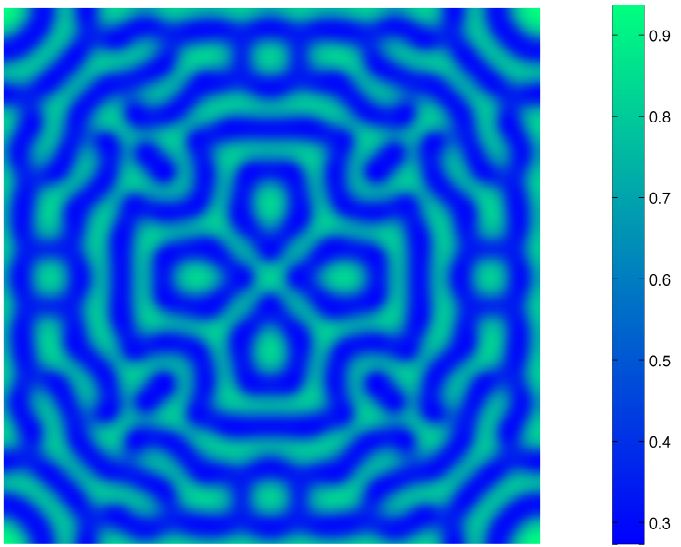

Solution at $\mathrm{t}=3000$ (First component) Parameters $(0.16,0.08,0.024349,0.081349)$, Noise $(0.001,0.001,2)$

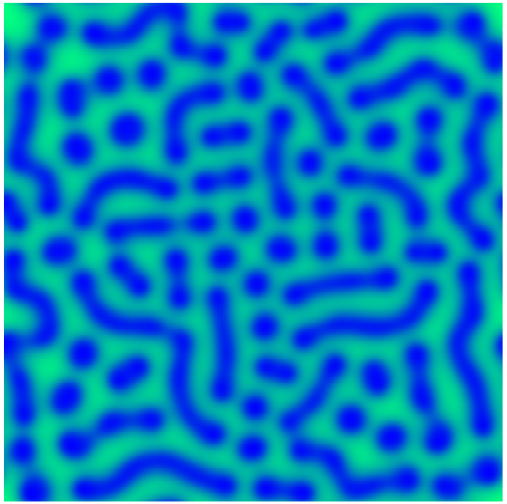

Solution at $\mathrm{t}=3000$ (First component)

Parameters $(0.16,0.08,0.029,0.086)$, Noise $(0.001,0.001,2)$

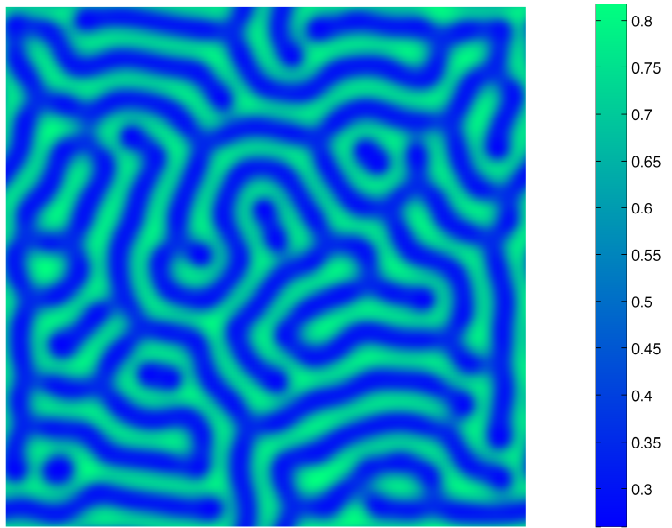

Figure 3: $\quad$ Stochastic Gray-Scott equations with parameters $\left(r_{u}, r_{v}, \widetilde{\alpha}_{u}, \widetilde{\alpha}_{v}, \sigma_{u}, \sigma_{v}, \gamma\right)$. First component of numerical solution at two times. available at http://techmath.uibk.ac.at/mecht/MyHomepage/Research/MovieMyCase3.mov http://techmath.uibk.ac.at/mecht/MyHomepage/Research/MovieMyCase31.mov http://techmath.uibk.ac.at/mecht/MyHomepage/Research/MovieMyCase32.mov 
is given as the limit of the infinite series

$$
\sum_{m \in \mathbb{N}^{d}} \int_{0}^{t} Y(\omega, s) h_{m} \mathrm{~d}\left(W(\omega, s) \mid h_{m}\right)_{\mathcal{H}}
$$

in $L^{2}(\Omega, \widetilde{\mathcal{K}})$ and leads to a well-defined continuous square-integrable martingale in $\widetilde{\mathcal{K}}$. In addition, fundamental results such as the Itô isometry

$$
\mathbb{E}\|J(T)\|_{\tilde{\mathcal{K}}}^{2}=\mathbb{E}\|Y\|_{L^{2}\left([0, T], L_{\mathrm{HS}}(\mathcal{H}, \widetilde{\mathcal{K}})\right)}^{2},
$$

and the Burkholder-Davis-Gundy inequality for any $p \geq 1$

$$
\mathbb{E} \sup _{t \in[0, T]}\|J(t)\|_{\widetilde{\mathcal{K}}}^{p} \leq C_{p} \mathbb{E}\|Y\|_{L^{2}\left([0, T], L_{\mathrm{HS}}(\mathcal{H}, \widetilde{\mathcal{K}})\right)}^{p}, \quad p \in[1, \infty),
$$

are valid; in the context of bounded space domains, further auxiliary calculations concerning the Hilbert-Schmidt norm are given below.

In our application we are working on a bounded interval given by $[0,1]^{d}$, we consider our equation on the $d$ dimensional torus. In the case of a single dimension, a complete orthonormal system of the underlying Lebesgue space $L^{2}(I):=L^{2}(I, \mathbb{R})$ is given by sine and cosine functions

$$
\psi_{m}(x)= \begin{cases}\sqrt{2} \sin (2 \pi m x) & \text { if } m \geq 1 \\ \sqrt{2} & \text { if } m=0 \\ \sqrt{2} \cos (\pi 2 m x) & \text { if } m \leq-1\end{cases}
$$

The extension to higher space dimensions relies on tensor products, i.e., for a multiindex $m=$ $\left(m_{1}, \ldots, m_{d}\right) \in \mathbb{Z}^{d}$ we have

$$
\phi_{m}(x)=\prod_{j=1}^{d} \phi_{m_{j}}\left(x_{j}\right), \quad x \in I .
$$

The corresponding eigenvalues are given by

$$
\lambda_{m}=-4 \pi^{2} \sum_{j=1}^{d} m_{j}^{2}, \quad m=\left(m_{1}, \ldots, m_{d}\right) \in \mathbb{Z}^{d} .
$$

The Wiener process $W$ has the following representation

$$
W(t, x)=\sum_{k \in \mathbb{Z}^{d}}\left(\alpha-D \lambda_{k}\right)^{-\gamma} \psi_{k}(x) \beta_{k}(t), \quad j=1,2,
$$


with $\gamma>\frac{d}{2}$ and where $\left\{\beta_{k}: k \in \mathbb{Z}^{d}\right\}$ is a family of one-dimensional Brownian motions. Let $\mathcal{H}:=L^{2}(I)$ and let $Q$ be defined by

$$
Q\left(\psi_{k}, \psi_{l}\right)=\delta_{k, l}\left(\alpha-D \lambda_{k}\right)^{-\gamma}, \quad k, l \in \mathbb{Z} .
$$

The covariance operator $Q$ can be expressed by the norm in Sobolev spaces. To do this, we relate the inner product and the associated norm to fractional Laplace operators

$$
\begin{gathered}
\left(\phi_{1} \mid \phi_{2}\right)_{W_{2}^{\kappa}(I)}=\left((\alpha-D \Delta)^{\kappa} \phi_{1} \mid \phi_{2}\right)_{L^{2}}, \\
\|\phi\|_{W_{2}^{\kappa}}=\left\|(\alpha-D \Delta)^{\frac{\kappa}{2}} \phi\right\|_{L^{2}}, \\
\phi, \phi_{1}, \phi_{2} \in W_{2}^{\kappa}(I), \quad \alpha>0, \quad D>0, \quad \kappa \in \mathbb{R} .
\end{gathered}
$$

Due to the fact that different choices of $\alpha>0$ and $D>0$ lead to equivalent norms, we only indicate the dependence on the decisive exponent $\kappa \in \mathbb{R}$. For scaled Fourier functions, we henceforth employ the abbreviation

$$
\psi_{m}^{(\kappa)}=\left(\alpha-D \lambda_{m}\right)^{-\frac{\kappa}{2}} \psi_{m}, \quad m \in \mathbb{Z}^{d},
$$

which permits to significantly reduce the length of formulas; here, we again suppress the dependence on $\alpha>0$ and $D>0$. The eigenvalue relation

$$
(\alpha-D \Delta)^{\frac{\kappa}{2}} \psi_{m}=\left(\alpha-D \lambda_{m}\right)^{\frac{\kappa}{2}} \psi_{m}, \quad m \in \mathbb{Z}^{d},
$$

implies that $\left(\psi_{m}^{(\kappa)}\right)_{m \in \mathbb{Z}^{d}}$ forms a complete orthonormal system of the fractional Sobolev space $H_{2}^{\kappa}(I)$, that is

$$
\left(\psi_{\ell}^{(\kappa)} \mid \psi_{m}^{(\kappa)}\right)_{W_{2}^{\kappa}(I)}=\delta_{\ell m}, \quad \ell, m \in \mathbb{Z}^{d} .
$$

The operator $Q$ is given as

$$
Q \psi_{m}\left(\psi_{k}=\delta_{k, m}\left(\alpha-D \lambda_{m}\right)^{-\gamma} .\right.
$$

\section{Appendix A.1. The multiplication operator}

In our equation, the diffusion coefficient in front of the stochastic perturbation is given by the multiplication operator defined by a function $\phi$, which is interpreted as a mapping from the Hilbert space $\mathcal{H}$ to the other Hilbert space $L^{2}(I)$. To be more precise,

$$
M(\phi): \mathcal{H} \longrightarrow \mathcal{K}: \chi \longmapsto \phi \chi,
$$

which complies with [38, Eq. (1.4)]; within the article, however, we often wrote

$$
\phi=M(\phi)
$$


for short. Let $\gamma>\frac{d}{2}$ and $\mathcal{H}=H_{2}^{\gamma}(I)$. Besides, let us denote the orthonormal basis in $\mathcal{H}$ by $\left\{\psi_{m}^{(\gamma)}: m \in \mathbb{Z}\right\}$, which is given by

$$
\psi_{m}^{(\gamma)}=\left(\alpha-D \lambda_{m}\right)^{-\frac{\gamma}{2}} \psi_{m}, \quad m \in \mathbb{Z}^{d}
$$

Then arguments detailed below show that for the particular case

$$
M(\phi): \mathcal{H} \longrightarrow L^{2}(I): \chi \longmapsto \phi \chi, \quad \phi \in L^{2}(I), \quad \gamma>\frac{d}{2}
$$

the associated Hilbert-Schmidt norm is finite, since the estimate

$$
\|M(\phi)\|_{L_{\mathrm{HS}}\left(\mathcal{H}, L^{2}\right)}=\left(\sum_{m \in \mathbb{Z}^{d}}\left\|\phi \psi_{m}^{(\gamma)}\right\|_{L^{2}}^{2}\right)^{\frac{1}{2}} \leq C \sqrt{S(\gamma)}\|\phi\|_{L^{2}}, \quad \gamma>\frac{d}{2},
$$

holds. Indeed, the stated bound is obtained from a representation of the defining real-valued function with respect to the complex-valued Fourier functions

$$
\phi=\sum_{\ell \in \mathbb{Z}^{d}}\left(\phi \mid \psi_{\ell}\right)_{L^{2}} \psi_{\ell}
$$

Let $\gamma>\frac{d}{2}$ and $\mathcal{H}=H_{2}^{\gamma}(I)$. Besides, let us denote the orthonormal basis in $\mathcal{H}$ by $\left\{\psi_{m}^{(\gamma)}\right.$ : $m \in \mathbb{Z}\}$, which is given by

$$
\psi_{m}^{(\gamma)}=\left(\alpha-D \lambda_{m}\right)^{-\frac{\gamma}{2}} \psi_{m}, \quad m \in \mathbb{Z}^{d},
$$

The associated Hilbert-Schmidt norm is finite. To be more precise, since the estimate

$$
\|M(\phi)\|_{L_{\mathrm{HS}}\left(\mathcal{H}, L^{2}\right)}=\left(\sum_{m \in \mathbb{Z}^{d}}\left\|\phi Q^{\frac{1}{2}} \psi_{m}\right\|_{L^{2}}^{2}\right)^{\frac{1}{2}} \leq C \sqrt{S(\gamma)}\|\phi\|_{L^{2}}, \quad \gamma>\frac{d}{2},
$$

holds. What happens if the underlying Hilbert space is $H_{2}^{\rho}(I)$ instead of $L^{2}(I)$.

We can write $\phi$ in terms of the orthonormal basis, i.e.

$$
\phi=\sum_{\ell \in \mathbb{Z}^{d}}\left(\phi \mid \psi_{\ell}\right)_{L^{2}} \psi_{\ell}
$$

In case, the underlying space is $H_{2}^{\delta}(I)$, we get

$$
\|M(\phi)\|_{L_{\mathrm{HS}}\left(\mathcal{H}, L^{2}\right)}=\left(\sum_{m \in \mathbb{Z}^{d}}\left\|\phi Q^{\frac{1}{2}} \psi_{m}\right\|_{L^{2}}^{2}\right)^{\frac{1}{2}} \leq C \sqrt{S(\gamma)}\|\phi\|_{L^{2}}, \quad \gamma>\frac{d}{2}
$$


By simple calculations, the following identity can be show:

$\psi_{m} \psi_{k}=\frac{1}{2} \begin{cases}=\left(\psi_{-|k-m|}-\psi_{-(k+m)}\right)=\left(\psi_{-|k-m|}-\psi_{-|k+m|}\right) & \text { for } m, k \geq 1, \\ \left(\psi_{|m|+|k|}-\psi_{|| k|-| m||}\right) & \text { for } k \geq 1, m \leq-1, \text { or, } m \geq 1, k \leq-1, \\ \left(\psi_{-|k-m|}-\psi_{-|k+m|}\right)=\left(\psi_{-|k-m|}-\psi_{-(k+m)}\right) & \text { for } m, k \leq-1, \\ 2 \psi_{m} & \text { for } m \in \mathbb{Z}, k=0,\end{cases}$

Using this identity, we obtain for $m \geq 1$ and $d=1$

$$
\begin{aligned}
& \phi \psi_{m} \\
= & \left(\phi \mid \psi_{0}\right)_{L^{2}}+\sum_{k \in \mathbb{N}}\left(\psi_{-|k-m|}-\psi_{-|k+m|}\right)\left(\phi \mid \psi_{k}\right)_{L^{2}}+\sum_{k \in \mathbb{N}}\left(\psi_{|k|+|m|}-\psi_{|| m|-| k||}\right)\left(\phi \mid \psi_{-k}\right)_{L^{2}} .
\end{aligned}
$$

Similarly, we get for $m \leq-1$ and $d=1$

$$
\begin{aligned}
& \phi \psi_{-|m|} \\
= & \left(\phi \mid \psi_{0}\right)_{L^{2}}+\sum_{k \in \mathbb{N}}\left(\psi_{-|k-m|}-\psi_{-|k+m|}\right)\left(\phi \mid \psi_{-k}\right)_{L^{2}}+\sum_{k \in \mathbb{N}}\left(\psi_{|k|+|m|}-\psi_{|| m|-| k||}\right)\left(\phi \mid \psi_{k}\right)_{L^{2}} \\
= & \left(\phi \mid \psi_{0}\right)_{L^{2}}+\sum_{k \in \mathbb{N}}\left(\psi_{-|k+| m||}-\psi_{-|k-| m||}\right)\left(\phi \mid \psi_{-k}\right)_{L^{2}}+\sum_{k \in \mathbb{N}}\left(\psi_{|k|+|m|}-\psi_{|| m|-| k||}\right)\left(\phi \mid \psi_{k}\right)_{L^{2}} .
\end{aligned}
$$

Evaluating carefully the $L^{2}(I)$-norm, we get for any $m \in \mathbb{Z}^{d}$

$$
\left|\phi \psi_{m}\right|_{L^{2}}^{2} \leq 4 \sum_{k \in \mathbb{Z}^{d}}\left(\phi \mid \psi_{k}\right)_{L^{2}} .
$$

Hence, we get for the trace

$$
\|M(\phi)\|_{L_{\mathrm{HS}}\left(\mathcal{H}, L^{2}\right)}=\left(\sum_{m \in \mathbb{Z}^{d}}\left\|\phi \psi_{m}^{(\gamma)}\right\|_{L^{2}}^{2}\right)^{\frac{1}{2}} \leq C \sqrt{S(\gamma)}\|\phi\|_{L^{2}}, \quad \gamma>\frac{d}{2},
$$

$\psi_{\ell} \psi_{m}=(2 a)^{-\frac{d}{2}} \psi_{\ell+m}$, we have

$$
\begin{aligned}
& \phi \psi_{m}^{(\gamma)}=\sum_{\ell \in \mathbb{Z}^{d}}\left(\phi \mid \psi_{\ell}\right)_{L^{2}} \psi_{\ell} \psi_{m}^{(\gamma)} \\
& \quad=(2 a)^{-\frac{d}{2}} \sum_{\ell \in \mathbb{Z}^{d}}\left(\alpha-D \lambda_{m}\right)^{-\frac{\gamma}{2}}\left(\phi \mid \psi_{\ell}\right)_{L^{2}} \psi_{\ell+m}, \quad m \in \mathbb{Z}^{d} .
\end{aligned}
$$


July 25, 2019

Parseval's identity, summation, and an integrability criterium for infinite series confirms the given result

$$
\begin{aligned}
& \|M(\phi)\|_{L_{\mathrm{HS}}\left(\mathcal{H}, L^{2}\right)}^{2}=\sum_{m \in \mathbb{Z}^{d}}\left\|\phi \psi_{m}^{(\gamma)}\right\|_{L^{2}}^{2} \\
& \quad=(2 a)^{-d}\left(\sum_{\ell \in \mathbb{Z}^{d}}\left|\left(\phi \mid \psi_{\ell}\right)_{L^{2}}\right|^{2}\right)\left(\sum_{m \in \mathbb{Z}^{d}}\left(\alpha-D \lambda_{m}\right)^{-\gamma}\right) \\
& \quad \leq C S(\gamma)\|\phi\|_{L^{2}}^{2} .
\end{aligned}
$$

By the very same calculation, one can show that we have

$$
\|M(\phi)\|_{L_{\mathrm{HS}}\left(\mathcal{H}, H_{2}^{1}(I)\right)} \leq C \sqrt{S(\gamma)}\|\phi\|_{H_{2}^{1}(I)}, \quad \gamma>\frac{d}{2},
$$

Let us denote for a Hilbert space $H$ the space of progressively measurable processes

$$
Y: \Omega \times[0, T] \rightarrow L_{H S}(\mathcal{H}, H)
$$

such that

$$
\mathbb{E}|Y|_{L^{2}\left([0, T] ; L_{H S}(\mathcal{H}, H)\right)}<\infty
$$

by $M_{\mathcal{H}}^{2}(0, T ; H)$. Having a process $Y \in M_{\mathcal{H}}^{2}\left(0, T ; L^{2}(\mathbb{R})\right)$ we get

$$
\mathbb{E} \sup _{t \in[0, T]}\left|\int_{0}^{t} M(Y(s)) d W(s)\right|_{L^{2}}^{p} \leq C_{p} S(\gamma) \mathbb{E}\left(\int_{0}^{T}\|Y(t)\|_{L^{2}}^{2} d t\right)^{\frac{p}{2}}, \quad p \in[1, \infty),
$$

Similarly, we have

$$
\mathbb{E} \sup _{t \in[0, T]}\left|\int_{0}^{t} M(Y(s)) d W(s)\right|_{H_{2}^{1}}^{p} \leq C_{p} S(\gamma) \mathbb{E}\left(\int_{0}^{T}\|Y(t)\|_{H_{2}^{1}}^{2} d t\right)^{\frac{p}{2}}, \quad p \in[1, \infty),
$$

The Itô formula. Within the proof we apply the Itô formula for the function $\Phi(x)=|x|_{L^{p}}^{p}$, $p \geq 2$ to a given process driven by a Wiener process. The diffusion operator will be the multiplication operator defined in A.9). To be precise, let us put $\Phi(u)=\int_{I} u^{p}(x) d x$. Then $D \Phi(u)[h]=p \int_{I} u^{p-1}(x) h(x) d x$ and $D^{2} \Phi(u)\left[h^{1}, h^{2}\right]=p(p-1) \int_{I} u^{p-2}(x) h^{1}(x) h^{2}(x) d x$. The correction term in the Itô formula is now defined by

$\operatorname{Tr}\left[D^{2} \phi(\xi(s))\left[M(u(s)) Q^{\frac{1}{2}}\right]\left[M\left(u(s) Q^{\frac{1}{2}}\right]^{*}\right]=\frac{p(p-1)}{2} \sum_{k \in \mathbb{Z}} \int_{I} \xi(s, x)^{p-2}(x)\left[M(u) h_{k}\right](x)\left[M(u) h_{k}\right](x) d x\right.$. 
The definition of the multiplication operator gives

$$
\operatorname{Tr}\left[D^{2} \phi(\xi(s))\left[M(u(s)) Q^{\frac{1}{2}}\right]\left[M\left(u(s) Q^{\frac{1}{2}}\right]^{*}\right] \leq \frac{p(p-1)}{2} \sum_{k \in \mathbb{Z}} \int_{I}|\xi(s, x)|^{p-2}(x) u^{2}(x) h_{k}^{2}(x) d x\right.
$$

The Hölder inequality gives

$$
\operatorname{Tr}\left[D^{2} \phi(\xi(s))\left[M(u(s)) Q^{\frac{1}{2}}\right]\left[M\left(u(s) Q^{\frac{1}{2}}\right]^{*}\right] \leq \frac{p(p-1)}{2} S(\gamma) \int_{I}|\xi(s, x)|^{p-2}(x) u^{2}(x) d x .\right.
$$

In the case $\xi=u$ we get

$$
\operatorname{Tr}\left[D^{2} \phi(\xi(s))\left[M(u(s)) Q^{\frac{1}{2}}\right]\left[M\left(u(s) Q^{\frac{1}{2}}\right]^{*}\right] \leq \frac{p(p-1)}{2} S(\gamma)|u|_{L^{p}}^{p}\right.
$$

In the case, $\Phi(u)=\int_{I}(\nabla u)^{p}(x) d x$. Then

$$
\begin{aligned}
D \Phi(u)[h] & =p \int_{I}(\nabla u)^{p-1}(x) \nabla h(x) d x \text { and } \\
D^{2} \Phi(u)\left[h^{1}, h^{2}\right] & =p(p-1) \int_{I}(\nabla u)^{p-2}(x)\left(\nabla h^{1}\right)(x)\left(\nabla h^{2}\right)(x) d x
\end{aligned}
$$

we obtain

$$
\operatorname{Tr}\left[D^{2} \phi(\xi)\left[M(u(s)) Q^{\frac{1}{2}}\right]\left[M\left(u(s) Q^{\frac{1}{2}}\right]^{*}\right] \leq \frac{p(p-1)}{2} \sum_{k \in \mathbb{Z}} \int_{I}|\nabla \xi(s)|^{p-2}(x)\left[\nabla\left(u h_{k}\right)\right]^{2}(x) d x\right.
$$

Again, the Hölder inequality gives

$$
\begin{aligned}
& \operatorname{Tr}\left[D^{2} \phi(\xi)\left[M(u(s)) Q^{\frac{1}{2}}\right]\left[M\left(u(s) Q^{\frac{1}{2}}\right]^{*}\right]\right. \\
& \leq \frac{p(p-1)}{2}\left(S(\gamma) \int_{I}|\nabla \xi(s)|^{p-2}(x)[\nabla u]^{2}(x)+S(\gamma+1) \lambda_{k}, d x\right) \\
& \leq \frac{p(p-1)}{2} \sum_{k \in \mathbb{Z}}\left(\alpha-D \lambda_{k}\right)^{-2 \gamma} \int_{I}\left|u^{p}(x)\right| d x \leq \frac{p(p-1)}{2} S(\gamma)|u|_{L^{p}}^{p} .
\end{aligned}
$$


The Itô-Correction Term. Let us assume that the process $X$ solves an infinite dimensional differential equation driven given as follows:

$$
d X(t)=\Delta X(t) d t+\Sigma(X(t)) \circ d W(t), \quad X(0)=X_{0},
$$

where $\Delta$ denotes the Laplacian operator with periodic boundary conditions. As before, $\left\{\psi_{m}\right.$ : $\left.m \in \mathbb{Z}^{d}\right\}$ denotes the eigenfunctions of $\Delta$ and $\left\{\lambda_{m}: m \in \mathbb{Z}^{d}\right\}$ denotes the corresponding eigenvalues. In this way, the solution process $\xi$ of equation $A .25$ can be described by the SPDE given in terms of the Itô-integral by adding a correction term. The correction term can be calculated explicitly (see [15, p. 65, Section 4.5.1]), i.e., the equivalent Itô equation of A.25) is given by

$$
d \xi(t)=A \xi(t) d t+\frac{1}{2} D_{\xi} \sigma(\xi(t)) \sigma(\xi(t)) d t+\sigma(\xi(t)) d \mathcal{W}(t), \quad \xi(0)=\xi_{0} .
$$

Here, $D_{\xi}(\sigma(\xi))$ denotes the Frechet derivative of $\sigma$ with respect to $\xi$. In our case, the Wiener process is infinite dimensional, but can be written as a sum of infinitely many scalar Wiener processes with $\sum_{k \in \mathbb{Z}} \sigma_{k} d \beta_{k}(t)$, where $\left\{\beta_{k}: k \in \mathbb{Z}\right\}$ is a family of independent scalar valued Wiener processes, and $\sigma_{k}$ is the multiplication operator given by $\xi \psi_{k} \lambda_{k}$. Straightforward calculations reveal

$$
\sum_{k \in \mathbb{Z}} D_{\xi} \sigma_{k}(\xi) \sigma_{k}(\xi)=\sum_{k \in \mathbb{Z}} \xi \psi_{k}^{2} \lambda_{k}^{2}
$$

Taking into account that $\lambda_{k}=\lambda_{-k}$ and $\psi_{k}^{2}+\psi_{-k}^{2}=2$, we have

$$
\sum_{k \in \mathbb{Z}} D_{\xi}\left(\sigma_{k}(\xi)\right) \sigma_{k}(\xi)=\gamma \xi
$$

where $\gamma=\sum_{k \in \mathbb{Z}} \lambda_{k}^{2}$.

\section{References}

[1] L. Arnold. Stochastic Differential Equations. Wiley and Suns, New York, 1974.

[2] L. Arnold, G. Bleckert, K. R. Schenk-Hoppé. The Stochastic Brusselator: Parametric Noise Destroys Hopf Bifurcation. In Stochastic Dynamics, Springer, New York, 1999.

[3] V. Barbu, G. Da Prato, and R. Röckner. Stochastic porous media equations. Lecture Notes in Mathematics 2163. Cham: Springer (2016).

[4] V. Barbu and M. Röckner. A splitting algorithm for stochastic partial differential equations driven by linear multiplicative noise. Stoch. Partial Differ. Equ. Anal. Comput., 5(4):457-471, 2017. 
[5] C. Bauzet, J. Charrier, and T. Gallouët. Convergence of flux-splitting finite volume schemes for hyperbolic scalar conservation laws with a multiplicative stochastic perturbation. Math. Comp., 85(302):2777-2813, 2016.

[6] C. Bauzet. On a time-splitting method for a scalar conservation law with a multiplicative stochastic perturbation and numerical experiments. J. Evol. Equ., 14(2):333-356, 2014.

[7] H. Bessaih, Z. Brzeźniak, and A. Millet. Splitting up method for the 2D stochastic NavierStokes equations. Stoch. Partial Differ. Equ. Anal. Comput., 2(4):433-470, 2014.

[8] C-E. Bréhier, G. Goudenège. Analysis of some splitting schemes for the stochastic AllenCahn equation, Discrete and Continous Dynamical Systems, Series B, available online (June 2019).

[9] T. Biancalani, D. Fanelli, F. Di Patti. Stochastic Turing patterns in the Brusselator model. Phys Rev E 81 (2010) 046215.

[10] Y. Cao, R. Erban. Stochastic Turing patterns: analysis of compartment-based approaches. Bull Math Biol 76 (2014) 3051-3069.

[11] E. Carelli, E. Hausenblas, and A. Prohl. Time-splitting methods to solve the stochastic incompressible Stokes equation. SIAM J. Numer. Anal., 50(6):2917-2939, 2012.

[12] P. L. Chow. Stochastic Partial Differential Equations. Second edition, Advances in Applied Mathematics, CRC Press, Boca Raton, FL, 2014.

[13] R. Dalang. Extending martingale measure stochastic integral with applications to spatially homogeneous SPDE's. Electron. J. Probab. 4/6 (1999) 1-29.

[14] G. Da Prato, J. Zabczyk. Stochastic Equations in Infinite Dimensions. Cambridge University Press, 2014.

[15] J. Duan, W. Wang. Effective dynamics of stochastic partial differential equations. Elsevier Insights. Elsevier, Amsterdam, 2014.

[16] L.C. Evans An Introduction to Stochastic Differential Equations AMS, 2013.

[17] M. Ferrante, M. Sanz-Solé. SPDEs with coloured noise: analytic and stochastic approaches. ESAIM Probab. Stat. 10 (2006) 380-405.

[18] P. Gray, S. K. Scott. Autocatalytic reactions in the isothermal continuous stirred tank reactor: isolas and other forms of multistability. Chem. Eng. Sci. 38 (1983) 29-43. 
[19] P. Gray, S. K. Scott. Autocatalytic reactions in the isothermal continuous stirred tank reactor: oscillations and instabilities in the system $a+2 b \rightarrow 3 b, b \rightarrow c$. Chem. Eng. Sci. 39 (1984) 1087-1097.

[20] P. Gray, S.K. Scott. Sustained oscillations and other exotic patterns of behaviour in isothermal reactions. J. Phys. Chem. 89/1 (1985) 22-32.

[21] P. Gray, S. K. Scott. Chemical Oscillations and Instabilities. Non-linear Chemical Kinetics. Clarendon Press, Oxford, 1994.

[22] E. Hairer, Ch. Lubich, G. Wanner. Geometric Numerical Integration. Structure-Preserving Algorithms for Ordinary Differential Equations. Second edition, Springer, Berlin, 2002.

[23] M. Hochbruck and A. Ostermann. Exponential integrators. Acta Numer., 19:209-286, 2010 .

[24] K. H. Karlsen and E. B. Storrø sten. Analysis of a splitting method for stochastic balance laws. IMA J. Numer. Anal., 38(1):1-56, 2018.

[25] H. Kierstead, L.B. Slobodkin. The size of water masses containing plankton blooms. Journal of Marine Research 12 (1953) 141-147.

[26] C. A. Klausmeier. Regular and irregular patterns in semi-arid vegetation. Science 284 (1999) 1826-1828.

[27] P. Kotelenez. Comparison methods for a class of function valued stochastic partial differential equations. Probab. Theory Relat. Fields 93 (1992) 1-19.

[28] R. Lefever, I. Prigogine. Symmetry breaking instabilities in dissipative systems II. J. Chem. Phys. 48 (1968) 1695-1700.

[29] S. A. Levin, L. A. Segel. Hypothesis for origin of planktonic patchiness. Nature 259 (1976) 659.

[30] W. Liu, M. Röckner. Stochastic Partial Differential Equations: An Introduction. Universitext, Springer, Cham, 2015.

[31] A. Lodhia, S. Sheffield, X. Sun, S.S. Watson. Fractional Gaussian fields: a survey. Probab. Surv. 13 (2016) 1-56.

[32] G. Lord, J.A. Rougemont. A numerical scheme for stochastic PDEs with Gevrey regularity. IMA J. Numer. Anal. 24 (2004), 587-604. 
[33] G. Lord, J.A. Rougemont. Topological and $\epsilon$-entropy for large volume limits of discretized parabolic equations, SIAM J. Numer. Anal. 40, (2002), 1311-1329.

[34] A. J. McKane, T. Biancalani, T. Rogers. Stochastic pattern formation and spontaneous polarisation: the linear noise approximation and beyond. Bull Math Biol. 76/4 (2014) 895-921.

[35] J. D. Murray. Biological pattern formation - a marriage of theory and experiment. In Mathematical Models for Biological Pattern Formation, Springer, New York, 2000, 1-9.

[36] J. D. Murray. Mathematical Biology II. Spatial Models and Biomedical Applications. Third edition, Springer, New York, 2003.

[37] D. Nualart. Fractional Brownian motion: stochastic calculus and applications. In Proceedings of the International Congress of Mathematicians, European Mathematical Society, Madrid, Spain, 2006, 1541-1562.

[38] S. Peszat, J. Zabczyk. Stochastic evolution equations with a spatially homogeneous Wiener process. Stochastic Processes Appl. 72 (1997) 187-204.

[39] D. Puhst. Gebrochene Ableitungen und Anwendungen. Bachelorarbeit, TU Berlin, 2010.

[40] M. Sango. Splitting-up scheme for nonlinear stochastic hyperbolic equations. Forum Math., 25(5):931-965, 2013.

[41] M. Scheutzow. Periodic behavior of the stochastic Brusselator in the mean-field limit. Probab. Theory Relat. Fields 72 (1986) 425-462.

[42] L. A. Segel, J. L. Jackson. Dissipative structure: An explanation and an ecological example. J. Thero. Biol. 37 (1972) 545-592.

[43] S. Sheffield. Gaussian free fields for mathematicians. Probab. Theory Relat. Fields 139 (2007) 521-541.

[44] P. Takáč, A. Jüngel. A nonstiff Euler discretization of the complex Ginzburg-Landau equation in one space dimension, SIAM J. Numer. Anal. 38, (2000), 292-328.

[45] G. Tessitore, J. Zabczyk. Strict positivity for stochastic heat equations. Stochastic Processes Appl. 77 (1998) 83-98.

[46] M. Thalhammer. Convergence analysis of high-order time-splitting pseudo-spectral methods for nonlinear Schrödinger equations. SIAM J. Numer. Anal. 50/6 (2012) 3231-3258. 
[47] M. Thalhammer, J. Abhau. A numerical study of adaptive space and time discretizations for Gross-Pitaevskii equations. J. Comput. Phys. 231 (2012) 6665-6681.

[48] J. Tu, Y. You. Random attractor of stochastic Brusselator system with multiplicative noise. Discrete Contin. Dyn. Syst. 36/5 (2016) 2757-2779.

[49] A. M. Turing. The chemical basis of morphogenesis. Phil. Trans. Roy. Soc. Lond. B 237/641 (1952) 37-72.

[50] J. B. Walsh. An introduction to stochastic partial differential equations. In École d'été de probabilités de Saint Flour XIV, Lecture Notes in Mathematics, Springer, Berlin, 1984, 265-439. 